\title{
Open Rectangle-of-Influence Drawings of Inner Triangulated Plane Graphs
}

\author{
Kazuyuki Miura • Tetsuya Matsuno • \\ Takao Nishizeki
}

Received: 1 October 2007 / Revised: 28 May 2008 / Accepted: 30 May 2008 /

Published online: 4 July 2008

(C) Springer Science+Business Media, LLC 2008

\begin{abstract}
A straight-line drawing of a plane graph is called an open rectangle-ofinfluence drawing if there is no vertex in the proper inside of the axis-parallel rectangle defined by the two ends of every edge. In an inner triangulated plane graph, every inner face is a triangle although the outer face is not necessarily a triangle. In this paper, we first obtain a sufficient condition for an inner triangulated plane graph $G$ to have an open rectangle-of-influence drawing; the condition is expressed in terms of a labeling of angles of a subgraph of $G$. We then present an $O\left(n^{1.5} / \log n\right)$-time algorithm to examine whether $G$ satisfies the condition and, if so, construct an open rectangle-of-influence drawing of $G$ on an $(n-1) \times(n-1)$ integer grid, where $n$ is the number of vertices in $G$.
\end{abstract}

Keywords Graph drawing · Open rectangle-of-influence drawing · Inner triangulated plane graph

\section{Introduction}

Recently automatic aesthetic drawing of graphs has created intense interest due to their broad applications, and as a consequence, a number of drawing methods have come out [1,3-6, 15-18]. The most typical drawing of a plane graph $G$ is a straight-

K. Miura ( $₫)$

Faculty of Symbiotic Systems Science, Fukushima University, Fukushima 960-1296, Japan

e-mail: miura@ sss.fukushima-u.ac.jp

T. Matsuno $\cdot$ T. Nishizeki

Graduate School of Information Sciences, Tohoku University, Sendai 980-8579, Japan

T. Matsuno

e-mail: matsuno@nishizeki.ecei.tohoku.ac.jp

T. Nishizeki

e-mail: nishi@ecei.tohoku.ac.jp 


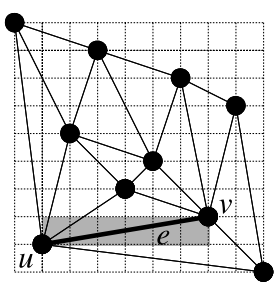

(a)

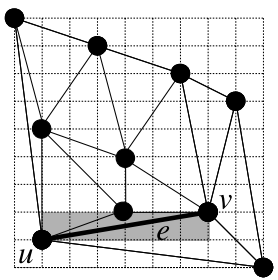

(b)

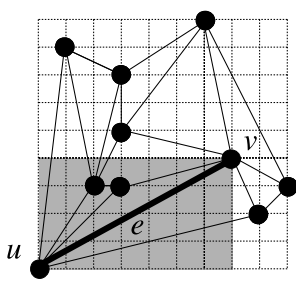

(c)

Fig. 1 (a) A closed RI-drawing, (b) an open RI-drawing, and (c) a non-RI-drawing of an inner triangulated plane graph without filled 3-cycles

line drawing, in which all vertices of $G$ are drawn as points and all edges are drawn as straight-line segments without any edge-intersection. A straight-line drawing is called a grid drawing if all vertices are put on grid points of integer coordinates. Figure 1 depicts three grid drawings of the same plane graph. A proximity drawing of a graph is a straight-line drawing where the points representing adjacent vertices are deemed to be close according to some proximity measure. Proximity drawings have been intensively studied in recent years because they arise in many areas including pattern recognition, geographic information systems, and computer vision [11].

In the paper, we deal with a type of a proximity drawing of a plane graph, known as a "rectangle-of-influence drawing" $[1,13]$. A rectangle-of-influence of an edge $e$ is an axis-parallel rectangle having $e$ as one of its diagonals. In each of Figs. 1(a)-(c), a rectangle-of-influence is shaded for an edge $e=(u, v)$ drawn by a thick line. We call a grid drawing a rectangle-of-influence drawing (or simply an RI-drawing) if there is no vertex in a rectangle-of-influence of any edge. Figures 1(a) and (b) depict RI-drawings, while Fig. 1(c) depicts a grid drawing which is not an RI-drawing. An RI-drawing often looks pretty, since vertices tend to be separated from edges. Our RI-drawing is the so-called weak RI-drawing [13]. A rectangle-of-influence of a line segment is defined similarly as that of an edge. A straight-line drawing is called a strong RI-drawing if there is an edge $(u, v)$ for every two vertices $u$ and $v$ such that no vertex is inside the rectangle-of-influence of segment $(u, v)$ [13]. Liotta et al. investigate whether several subclasses of planar graphs as well as complete graphs admit strong RI-drawings [13].

A rectangle-of-influence of an edge $e$ is closed if it contains the boundary of a rectangle and is open if it does not contain the boundary. In a closed RI-drawing, every rectangle-of-influence is regarded as a closed one, while in an open RI-drawing, every rectangle-of-influence is regarded as an open one. In a closed RI-drawing, there is no vertex except the ends not only in the proper inside of a rectangle-of-influence of each edge but also on the boundary, as illustrated in Fig. 1(a). In an open RIdrawing, there may be a vertex other than the ends on the boundary of a rectangle, as illustrated in Fig. 1(b). Thus a closed RI-drawing is an open RI-drawing, but an open RI-drawing is not always a closed RI-drawing.

Biedl et al. [1] showed that a plane graph $G$ has a closed RI-drawing if and only if $G$ has no filled 3-cycle, that is, a cycle of three vertices such that there is a vertex in the proper inside, and showed that $G$ has a closed RI-drawing on an $(n-1) \times(n-1)$ grid if $G$ has no filled 3-cycle, as illustrated in Fig. 1(a), where $n$ is the number of 


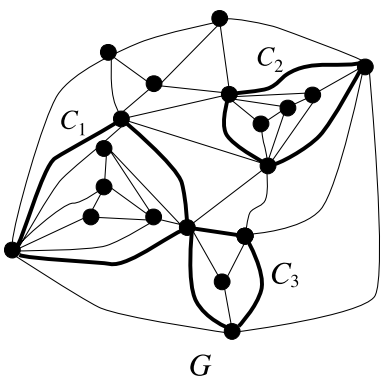

(a)

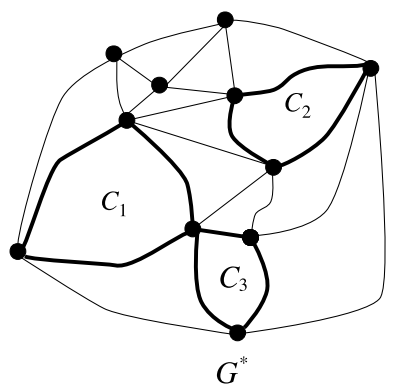

(c)

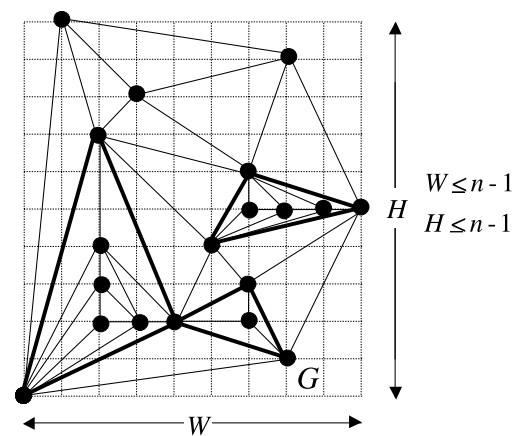

(b)

Fig. 2 (a) An inner triangulated plane graph $G$ with three maximal filled 3-cycles $C_{1}, C_{2}$, and $C_{3}$ drawn by thick lines, (b) an open RI-drawing of $G$, and (c) the frame graph $G^{*}$ of $G$

vertices in $G$. (The graph in Fig. 1 has no filled 3-cycle, while the graph in Fig. 2(a) has filled 3-cycles.) They also showed that one can test in linear time whether a given planar graph has a plane embedding without filled 3-cycles. It is also known that every 4-connected plane graph with four or more vertices on the outer facial cycle has an open RI-drawing on a smaller grid, that is, a $W \times H$ grid with $W+H \leq n$, and such a drawing can be found in linear time [15], where $W$ and $H$ are the width and height of an integer grid, respectively. A plane graph $G$ may have an open RIdrawing even if $G$ has a filled 3-cycle, as illustrated in Figs. 2(a) and (b). However, a necessary and sufficient condition for an open RI-drawing is not known. It is an open problem to characterize a class of plane graphs that have open RI-drawings [1] or strong open RI-drawings [13].

In a triangulated plane graph, all facial cycles are 3-cycles. In an inner triangulated plane graph, all inner facial cycles are 3-cycles although the outer facial cycle is not necessarily a 3-cycle, as illustrated in Fig. 2(a). Every plane graph can be augmented to a triangulated or inner triangulated plane graph under some constraint [2]. Some of the algorithms for drawing plane graphs first triangulate or inner-triangulate a given plane graph $G$ by adding dummy edges, find a drawing of the resulting graph, and finally erase the dummy edges from the drawing to obtain a drawing of $G$ $[1,6,16]$. 
Fig. 3 (a) A triangulated plane graph $G ;(\mathbf{b})$ an open RI-drawing $D$ of $G$

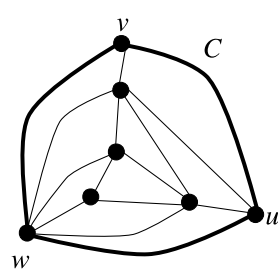

(a)

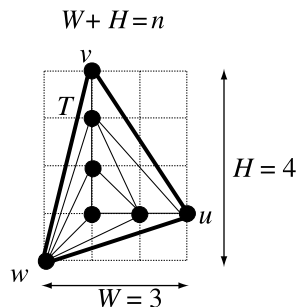

(b)

In the paper, we deal with open RI-drawings of triangulated plane graphs and inner triangulated plane graphs. We first show that one can decide in linear time whether a given triangulated plane graph $G$ has an open RI-drawing and that if $G$ has such a drawing, then it can be constructed in linear time on a $W \times H$ grid with $W+H=n$, where $n$ is the number of vertices in $G$ (see Fig. 3). We then obtain a sufficient condition for an inner triangulated plane graph $G$ to have an open RI-drawing (see Figs. 2(a) and (b)). Our condition is expressed in terms of a labeling of angles of a subgraph $G^{*}$ of $G$ with integers $0,1,2,3$, and 4 . The subgraph $G^{*}$, called the frame graph of $G$, is obtained from $G$ by removing all vertices and edges in the proper inside of every maximal filled 3-cycle of $G . G^{*}$ is an inner triangulated plane graph and has no filled 3-cycle, and hence $G^{*}$ has a closed RI-drawing. (Figure 2(c) depicts $G^{*}$ for $G$ in Fig. 2(a).) We show that $G$ has an open RI-drawing if $G^{*}$ has a kind of an angular labeling, called a "good labeling." We also present an $O\left(n^{1.5} / \log n\right)$ time algorithm to examine whether $G^{*}$ has a good labeling and, if so, construct an open RI-drawing of $G$ on an $(n-1) \times(n-1)$ grid from a good labeling of $G^{*}$. The complexity $O\left(n^{1.5} / \log n\right)$ is due to a step where the algorithm finds a perfect matching in a bipartite graph. An early version of the paper was presented at [14].

The remainder of the paper is organized as follows. In Sect. 2, we present some definitions and a known result. In Sect. 3, we show how to find an open RI-drawing of a triangulated plane graph. In Sect. 4, we first present a sufficient condition for an inner triangulated plane graph $G$ to have an open RI-drawing, that is, we claim that $G$ has an open RI-drawing if $G^{*}$ has a good labeling, and then present an algorithm to find an open RI-drawing of $G$ from a good labeling of $G^{*}$. In Sect. 5, we prove the sufficient condition. In Sect. 6, we present an algorithm to find a good labeling of $G^{*}$. Finally we conclude in Sect. 7 .

\section{Preliminaries}

In this section, we present some definitions and a known result.

We deal with an undirected simple graph $G$. The set of vertices in $G$ is denoted by $V(G)$. An edge joining vertices $u$ and $v$ is denoted by $(u, v)$. We denote by $u \rightarrow v$ the edge directed from $u$ to $v$. Throughout the paper, we denote by $n$ the number of vertices of a graph $G$. A $W \times H$ integer grid consists of $W+1$ vertical grid lines and $H+1$ horizontal grid lines. We call $W$ and $H$ the width and height of the integer grid, respectively. The $x$-coordinate of vertex $v$ is denoted by $x(v)$ and the $y$-coordinate by $y(v)$. 
A graph is planar if it can be embedded in the plane so that no two edges intersect geometrically except at a vertex to which they are both incident. A plane graph is a planar graph with a fixed embedding. A plane graph $G$ divides the plane into connected regions, called faces. The boundary of a face is called a facial cycle and is denoted by a sequence of the vertices on the boundary. The boundary of the outer face is called the outer facial cycle of $G$. A vertex on the outer facial cycle is called an outer vertex, while a vertex not on the outer facial cycle is called an inner vertex. An edge on the outer facial cycle is called an outer edge, while an edge not on the outer facial cycle is called an inner edge. A cycle of three vertices in $G$ is called a 3 -cycle. A 3-cycle of $G$ is filled if there is a vertex in the proper inside of $C$. The graph in Fig. 2(a) has three maximal filled 3-cycles $C_{1}, C_{2}$, and $C_{3}$, which are drawn by thick lines. A plane graph $G$ is inner triangulated if $G$ is 2-connected and every inner facial cycle is a 3-cycle.

A 3-cycle $C$ of a plane graph $G$ is drawn as a triangle $T$ in a grid drawing of $G$. Every triangle $T$ is covered by the three closed rectangle-of-influences of edges of $C$. Therefore, if $G$ has a closed RI-drawing, then $G$ has no filled 3-cycle. The converse is also true. Thus Biedl et al. obtained the following result [1].

Lemma 1 A plane graph $G$ has a closed RI-drawing if and only if $G$ has no filled 3 -cycle. Furthermore, $G$ has a closed RI-drawing on an $(n-1) \times(n-1)$ grid if $G$ has no filled 3-cycle.

A straight-line segment is oblique if it is neither horizontal nor vertical. Every edge is oblique in a closed RI-drawing obtained by the algorithm in [1]. A grid drawing of a plane graph is oblique if every edge is oblique in the drawing.

\section{Drawing Triangulated Plane Graphs}

Let $C$ be a maximal filled 3-cycle of an inner triangulated graph $G$. Then the inside graph $G(C)$ induced by all the vertices inside $C$ is a triangulated plane graph. (Figure 3(a) depicts $G\left(C_{1}\right)$ for $G$ and $C_{1}$ in Fig. 2(a).) In this section, we show how to find an open RI-drawing of an arbitrary triangulated graph.

Suppose that $G$ is an arbitrary triangulated plane graph with four or more vertices as illustrated in Fig. 3(a) and that $G$ has an open RI-drawing $D$ as illustrated in Fig. 3(b). The outer facial cycle $C=u v w$ of $G$ is filled and is drawn as a triangle $T$ in $D$. Two or three sides of the triangle $T$ are oblique; otherwise, $T$ has exactly one oblique side and $T$ is a right-angled triangle having both a vertical side and a horizontal side; the proper inside of such a triangle $T$ is covered by the open rectangle-of-influence of the oblique side, and hence the inner vertices of $G$ cannot be drawn. Thus there are the following three cases to consider.

(a) Two sides of $T$ are oblique, and the other side is horizontal, as illustrated in Fig. 4(a)

(b) Two sides of $T$ are oblique, and the other side is vertical, as illustrated in Fig. 4(b) and

(c) All the three sides of $T$ are oblique, as illustrated in Fig. 4(c). 


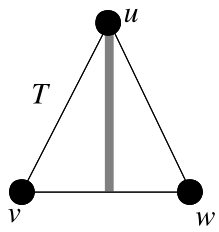

(a)

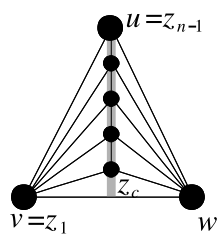

(d)

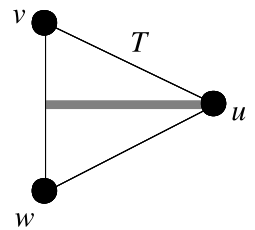

(b)

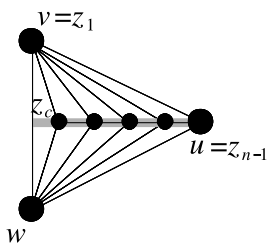

(e)

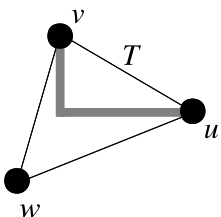

(c)

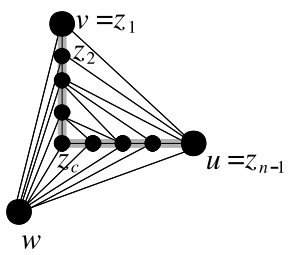

(f)

Fig. 4 (a)-(c) Three shapes of triangle $T ;(\mathbf{d})-(\mathbf{f})$ triangulated plane graphs $G$ and drawings $D$

Only the line segments in $T$ drawn by thick lines in Figs. 4(a)-(c) are not covered by the open rectangle-of-influences of three edges of $C$. Therefore, all inner vertices of $G$ must be located on the thick line segments in Figs. 4(a)-(c). Thus one can know that the graph $G$ and the drawing $D$ must have a structure illustrated in Fig. 4(f). More precisely, one of the three outer vertices $u, v$, and $w$ of $G$, say $w$, is adjacent to all the other vertices $z_{1}, z_{2}, \ldots, z_{n-1}$ in $G$. One may assume that $z_{1}=v, z_{n-1}=u$, and $z_{1}, z_{2}, \ldots, z_{n-1}$ is a path in the triangulated plane graph $G$. Then, for some index $c$, $2 \leq c \leq n-2$, every edge of $G$, that is neither incident to $w$ nor on the path above, joins vertices $z_{i}$ and $z_{j}$ with $1 \leq i<c<j \leq n-1$. The drawings in Figs. 4(d) and (e) are particular cases in which exactly two of the three outer vertices, say $v$ and $w$, are adjacent to all the other vertices in $G$, and hence $c=2$.

Conversely, if $G$ has a structure illustrated in Fig. 4(f), then $G$ has an open RIdrawing on a $W \times H$ grid such that $W+H=n$. Note that $W=n-c$ and $H=c$ for the index $c$ above.

We thus have the following theorem.

Theorem 1 One can decide in linear time whether a given triangulated plane graph $G$ has an open RI-drawing or not, and, if so, one can find in linear time an open RIdrawing of $G$ on a $W \times H$ grid such that $W+H=n$ and all the three outer edges are oblique.

One can enlarge the open RI-drawing of $G$ on a $W \times H$ grid such that $W+H=n$ to an open RI-drawing on an arbitrary $W^{\prime} \times H^{\prime}$ grid such that $W^{\prime} \geq W$ and $H^{\prime} \geq H$.

\section{Drawing Inner Triangulated Plane Graphs}

In Sect. 4.1, we describe a sufficient condition for an inner triangulated plane graph $G$ to have an open RI-drawing. (The condition will be verified in Sect. 5.) In Sect. 4.2, 
we give an algorithm to construct an open RI-drawing of $G$ if $G$ satisfies the condition.

\subsection{Sufficient Condition}

If $G$ has no filled 3-cycle, then by Lemma $1 G$ has a closed RI-drawing, which is an open RI-drawing. Therefore, we may assume without loss of generality that $G$ has filled 3-cycles. Let $C_{1}, C_{2}, \ldots, C_{k}, k \geq 1$, be the maximal filled 3-cycles of $G$. (The plane graph $G$ in Fig. 2(a) has three maximal filled 3-cycles $C_{1}, C_{2}$, and $C_{3}$ drawn by thick lines, and hence $k=3$.) The inside graph $G\left(C_{i}\right)$ induced by all the vertices inside $C_{i}$ is a triangulated plane graph. One may assume without loss of generality that $G\left(C_{i}\right)$ has an open RI-drawing for every index $i, 1 \leq i \leq k$; otherwise, $G$ has no open RI-drawing.

We will claim that if $G$ satisfies our sufficient condition, then $G$ has an open RIdrawing in which every edge of the frame graph $G^{*}$ of $G$ is oblique, as illustrated in Fig. 2(b). Assume that $G$ has an open RI-drawing $D$ in which every edge of $G^{*}$ is oblique. Thus, in $D$, every inside graph $G\left(C_{i}\right)$ is drawn so that all the three edges of $C_{i}$ are oblique, as illustrated in Fig. 4(f). An angle of $G^{*}$ is an angle of (a straight-line drawing of) a face of $G^{*}$. An angle of an inner face of $G^{*}$ is called an inner angle, while an angle of the outer face is called an outer angle. At each vertex $v$ in $G^{*}$, draw two lines, one with slope 0 and the other with slope $\infty$, as illustrated in Fig. 5 by dotted lines. These two lines define four half-lines at $v$. We say that an angle at $v$ contains a number $i$ of the four half-lines, $0 \leq i \leq 4$, if the region of the plane defined by that angle contains $i$ half-lines at $v$. (Note that there is no horizontal or vertical edge in $G^{*}$.) Thus, in Fig. 5, angles $\alpha_{0}, \alpha_{1}, \alpha_{2}$, and $\alpha_{3}$ contain $0,1,2$, and 1 halflines, respectively. In Fig. 6 , the outer angles of outer vertices $v_{i}, 0 \leq i \leq 4$, contain $i$ half-lines.

Our condition is expressed in terms of a labeling of the frame graph $G^{*}$ of $G$. A labeling $L^{*}$ of $G^{*}$ is an assignment of labels $0,1,2,3$, or 4 to each angle of $G^{*}$, as illustrated in Fig. 7(a). We say that an oblique drawing $D^{*}$ of $G^{*}$ realizes a labeling $L^{*}$ if every inner angle labeled $i$ by $L^{*}$ contains exactly $i$ half-lines in $D^{*}$ for each $i$,

Fig. 5 Angles $\alpha_{0}, \alpha_{1}, \alpha_{2}$, and $\alpha_{3}$

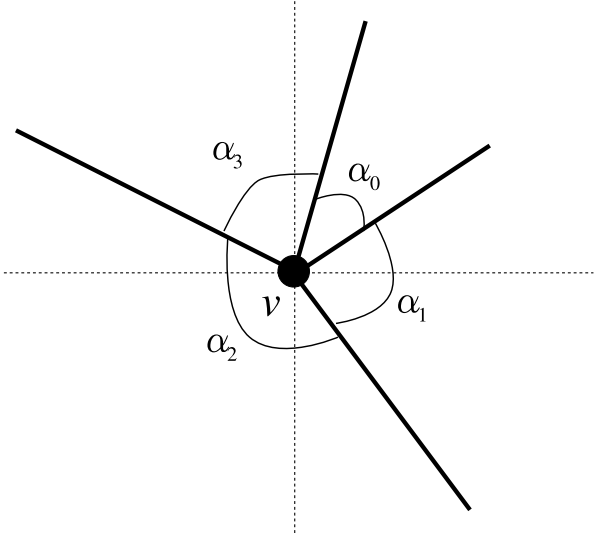


Fig. 6 Nonconvex outer facial cycle

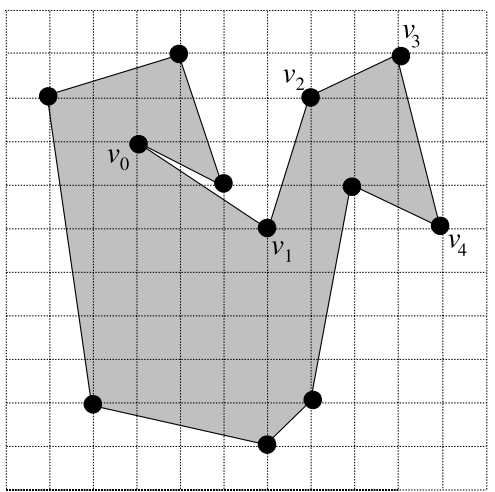

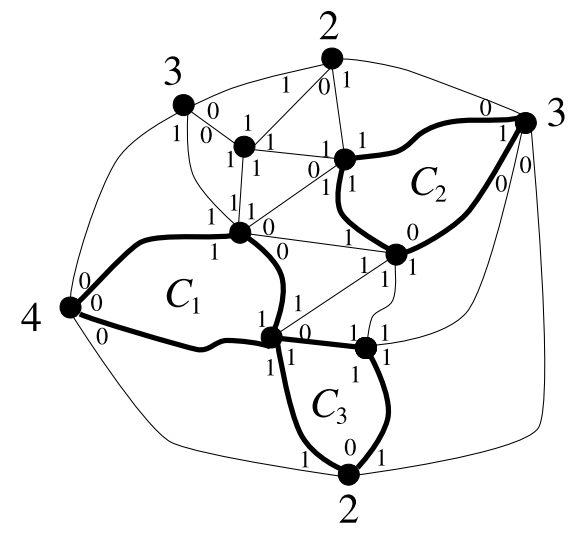

(a)

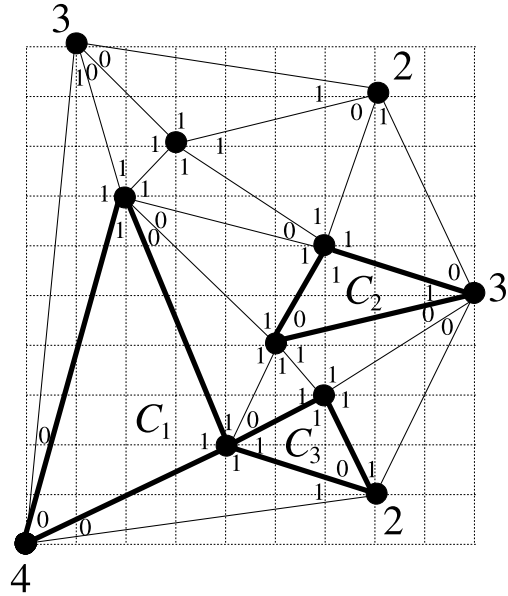

(b)

Fig. 7 (a) A good labeling $L^{*}$ of $G^{*}$, and (b) a good RI-drawing $D^{*}$ of $G^{*}$ realizing $L^{*}$

$0 \leq i \leq 4$. (The drawing in Fig. 7(b) realizes the labeling in Fig. 7(a).) For an oblique drawing $D^{*}$ of $G^{*}$, we denote by $L\left(D^{*}\right)$ the labeling of $G^{*}$ induced by $D^{*}$.

Suppose that an inner triangulated plane graph $G$ has an open RI-drawing $D$ in which every edge of $G^{*}$ is oblique. Let $D^{*}$ be a drawing of $G^{*}$ in $D$, then $D^{*}$ is oblique. Let $L\left(D^{*}\right)$ be a labeling of $G^{*}$ induced by $D^{*}$. Then, clearly $L\left(D^{*}\right)$ satisfies the following condition (a):

(a) For each vertex $v$ of $G^{*}$, the labels around $v$ total to 4 .

We now claim that $L\left(D^{*}\right)$ satisfies the following condition (b):

(b) Every inner facial 3-cycle $C$ of $G^{*}$ has labels 0,1 , and 1 . If $C$ is a maximal filled 3 -cycle in $G$, then the vertex labeled 0 in $C$ is adjacent to all the other vertices of the inside graph $G(C)$ of $C$. (See Fig. 8.) 
Fig. 8 Labelings of

(a) a non-filled 3-cycle $C$ and

(b) a filled 3-cycle $C$

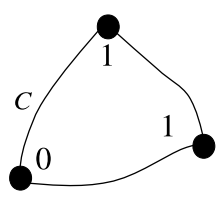

(a)

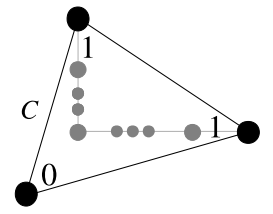

(b)

Fig. 9 A triangle such that an angle contain two half-lines

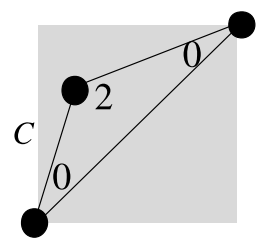

Since every edge of $G^{*}$ is oblique in $D^{*}$, every inner facial 3-cycle $C$ of $G^{*}$ is drawn as a triangle $T$ having three oblique sides. Clearly the three angles contain exactly two half-lines in total. Furthermore, two angles in $C$ contain exactly one half-line, and the other angle does not contain any half-line as illustrated in Fig. 8; if an angle in $C$ contains two half-lines, then the vertex of the angle would be in the proper inside of the rectangle-of-influence of the longest edge of $T$ as illustrated in Fig. 9. Thus $C$ has labels 0,1 , and 1 in the labeling $L\left(D^{*}\right)$. If $C$ is a maximal filled 3-cycle in $G$, then $G(C)$ is a triangulated plane graph and the vertex of $C$ labeled 0 must be adjacent to all the other vertices of $G(C)$, as illustrated in Fig. 4(f). We have thus proved that $L\left(D^{*}\right)$ satisfies condition (b).

Thus, if $G$ has an open RI-drawing in which every edge of $G^{*}$ is oblique, then $G^{*}$ has a labeling satisfying the two conditions (a) and (b) above. However, the converse is not necessarily true. For example, the plane graph $G$ in Fig. 10(a) has no open RIdrawing, but the frame graph $G^{*}$ has the unique labeling satisfying two conditions (a) and (b) depicted in Fig. 10(b).

In Sect. 4.2, we will show how to construct an open RI-drawing of $G$ from a labeling satisfying conditions (a), (b), and the following additional condition (c):

(c) Every outer angle has label 2, 3, or 4. (See Fig. 7.)

A labeling of $G^{*}$ satisfying three conditions (a)-(c) above is called a good labeling. (A good labeling has a close relation with the "regular edge-labeling" of Kant and He [12] and the "transversal structure" of Fusy [7] in the case where the outer face of $G^{*}$ is quadrangular.)

We claim the following theorem, the proof of which will be given in Sect. 5 .

Theorem 2 An inner triangulated plane graph $G$ has an open RI-drawing if the frame graph $G^{*}$ of $G$ has a good labeling.

One may prefer to draw the outer facial cycle of $G$ as a convex polygon for which each outer angle contains two, three, or four half-lines in $D$. We say that an RIdrawing $D$ of $G$ is good if every edge of $G^{*}$ is oblique and each outer angle contains 


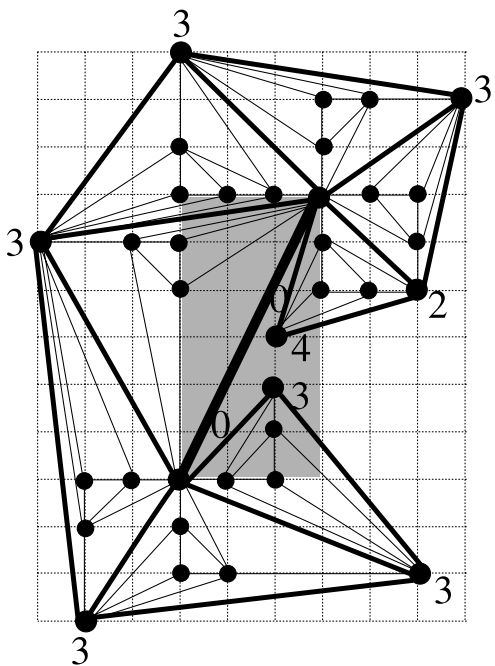

(a)

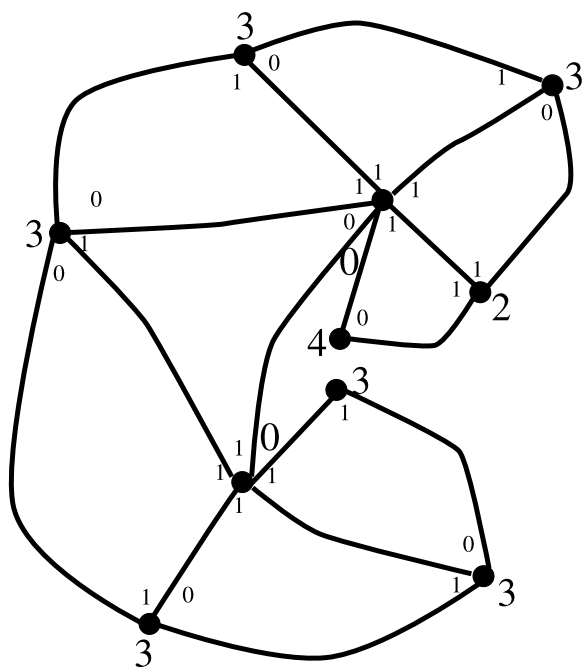

(b)

Fig. 10 (a) A plane graph $G$ having no open RI-drawing, and (b) the frame graph $G^{*}$ with a labeling satisfying conditions (a) and (b)

two, three, or four half-lines in $D$. For example, the drawings in Figs. 1(a), 1(b), 2(b), and 3(b) are good open RI-drawings, while an open RI-drawing having the nonconvex outer facial cycle in Fig. 6 is not good. It should be noted that the outer facial cycle of a good open RI-drawing is not necessarily a convex polygon, because there is a nonconvex polygon such that each outer angle contains two, three, or four half-lines. (See Figs. 22(a) and (c) in Sect. 5.) Indeed our result implies that

$G$ has a good open RI-drawing if and only if $G^{*}$ has a good labeling.

It is interesting to observe the following fact. When the maximal filled 3-cycles $C_{1}, C_{2}, \ldots, C_{k}$ are removed from an inner triangulated plane graph $G$, the resulting frame graph $G^{*}$ has no filled 3-cycle and hence, by the result in [1], one can construct a closed oblique RI-drawing $D^{*}$ of $G^{*}$. In order to construct an open RI-drawing of $G$, one has just to ensure that every maximal filled 3-cycle $C_{i}, 1 \leq i \leq k$, has a vertex $w$ such that $w$ is adjacent to all vertices inside $C_{i}$ and the angle incident to $w$ inside $C_{i}$ has no half-line in $D^{*}$, as illustrated in Fig. 4(f), that is, the angle has label 0 in a good labeling. Furthermore, the drawing of every inner face $F$ of $G^{*}$ in $D^{*}$ satisfies the following two conditions (i) and (ii):

(i) the three edges of $F$ cannot have the same slope with respect to the sign; and

(ii) among the three vertices of $F$, the vertex $w$ incident to an angle with label 0 must be either left-most or right-most and must be either top-most or bottom-most, as illustrated in Fig. 11.

\subsection{Constructing an Open RI-drawing from a Good Labeling}

Suppose that the frame graph $G^{*}$ of an inner triangulated plane graph $G$ has a good labeling $L^{*}$, as illustrated in Fig. 7(a). Remember that each inside graph $G\left(C_{i}\right), 1 \leq$ 
Fig. 11 Four types of a drawing of an inner face $F$ of $G^{*}$ and their orientation in $G_{x}^{*}$

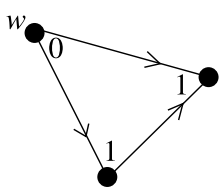

(a)

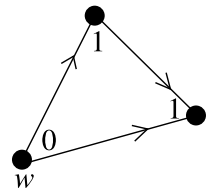

(c)

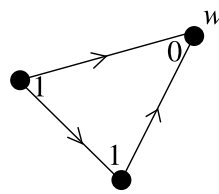

(b)

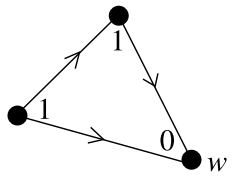

(d)

$i \leq k$, is assumed to have an open RI-drawing. We first obtain an open RI-drawing $D_{i}$ of each triangulated plane graph $G\left(C_{i}\right)$ as in Sect. 3. We then construct from $L^{*}$ an oblique RI-drawing $D^{*}$ of $G^{*}$ realizing $L^{*}$ so that every maximal filled cycle $C_{i}$, $1 \leq i \leq k$, has a vertex $w$ such that $w$ is adjacent to all vertices inside $C_{i}$ and the angle incident to $w$ inside $C_{i}$ has label 0 , as illustrated in Fig. 7(b). We finally embed in $D^{*}$ each drawing $D_{i}$ after adjusting the size of $D_{i}$ to the triangular drawing of $C_{i}$ in $D^{*}$, as illustrated in Fig. 2(b). (The drawing of $G\left(C_{1}\right)$ on a $3 \times 4$ grid in Fig. 3(b) is enlarged to a drawing on a $4 \times 7$ grid as illustrated in Fig. 2(b).) We claim that the resulting drawing $D$ is an open RI-drawing of $G$.

Our algorithm for constructing $D^{*}$ from $L^{*}$ consists of the following three steps.

Step 1 Directing each edge $(u, v)$ of $G^{*}$, we construct a directed graph $G_{x}^{*}$, as illustrated in Fig. 12(a); $(u, v)$ is directed from $u$ to $v$, that is, $u \rightarrow v$ if $x(u)<x(v)$ must hold in an open RI-drawing $D^{*}$ of $G^{*}$ realizing the labeling $L^{*}$, where $x(u)$ and $x(v)$ are the $x$-coordinates of vertices $u$ and $v$, respectively. Similarly, we construct a directed graph $G_{y}^{*}$, as illustrated in Fig. 12(b). More precisely, we construct $G_{x}^{*}$ and $G_{y}^{*}$ as follows.

Let $v_{1}$ and $v_{2}$ be any two outer vertices consecutively appearing clockwise on the outer facial cycle of $G^{*}$. A drawing obtained from an oblique RI-drawing $D^{*}$ of $G^{*}$ realizing $L^{*}$ by rotating it $90^{\circ}, 180^{\circ}$, or $270^{\circ}$ is also an oblique RI-drawing realizing $L^{*}$. Therefore, one may assume without loss of generality that $x\left(v_{1}\right)<$ $x\left(v_{2}\right)$ and $y\left(v_{1}\right)<y\left(v_{2}\right)$ in $D^{*}$. Let $F_{1}=v_{1} v_{2} v_{3}$ be the inner facial 3-cycle of $G^{*}$ having the edge $\left(v_{1}, v_{2}\right)$. Then the good labeling $L^{*}$ assigns label 0 to one of the three vertices $v_{1}, v_{2}$, and $v_{3}$ of $F_{1}$ and assigns label 1 to the other two vertices, as illustrated in Fig. 13. If $v_{1}$ has label 0 as in Fig. 13(a), then we decide that $x\left(v_{1}\right)<x\left(v_{2}\right)<x\left(v_{3}\right)$ and $y\left(v_{1}\right)<y\left(v_{3}\right)<y\left(v_{2}\right)$, and hence $v_{1} \rightarrow v_{2}, v_{1} \rightarrow v_{3}$, and $v_{2} \rightarrow v_{3}$ in $G_{x}^{*}$ and $v_{1} \rightarrow v_{2}, v_{1} \rightarrow v_{3}$, and $v_{3} \rightarrow v_{2}$ in $G_{y}^{*}$, as illustrated in Fig. 14(a). If $v_{2}$ has label 0 as in Fig. 13(b), then we decide that $v_{1} \rightarrow v_{2}, v_{1} \rightarrow v_{3}$, and $v_{3} \rightarrow v_{2}$ in $G_{x}^{*}$ and $v_{3} \rightarrow v_{1}$, $v_{3} \rightarrow v_{2}$, and $v_{1} \rightarrow v_{2}$ in $G_{y}^{*}$. If $v_{3}$ has label 0 as in Fig. 13(c), then we decide that $v_{1} \rightarrow v_{2}, v_{1} \rightarrow v_{3}$, and $v_{2} \rightarrow v_{3}$ in $G_{x}^{*}$ and $v_{3} \rightarrow v_{1}, v_{3} \rightarrow v_{2}$, and $v_{1} \rightarrow v_{2}$ in $G_{y}^{*}$. Thus we direct each edge of $F_{1}$ for $G_{x}^{*}$ and $G_{y}^{*}$. Note that the vertex incident to the angle in $F_{1}$ with label 0 has out-degree either 0 or 2 on $F_{1}$ in $G_{x}^{*}$ and $G_{y}^{*}$. We then 


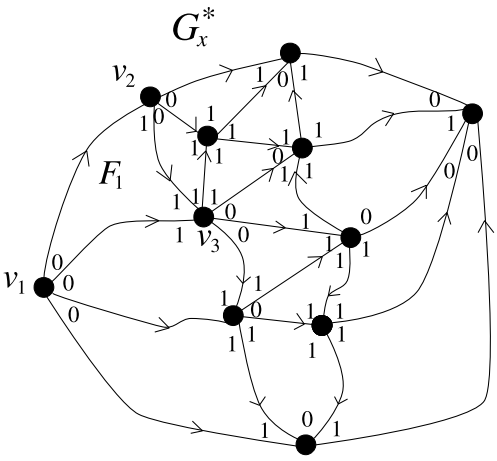

(a)

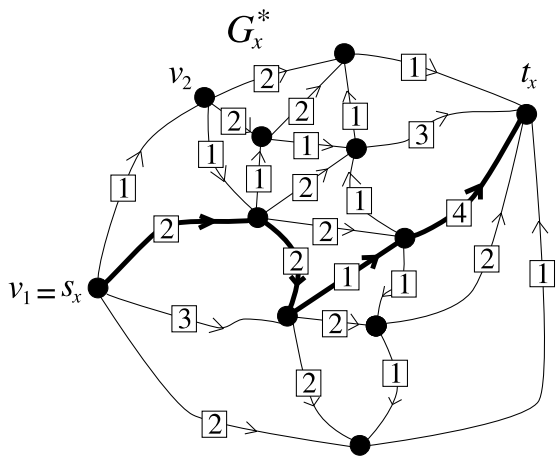

(c)

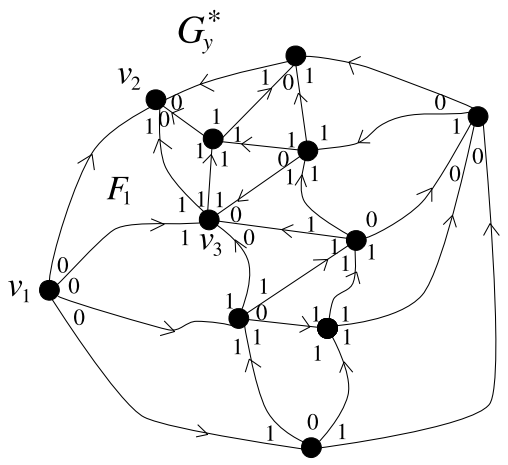

(b)

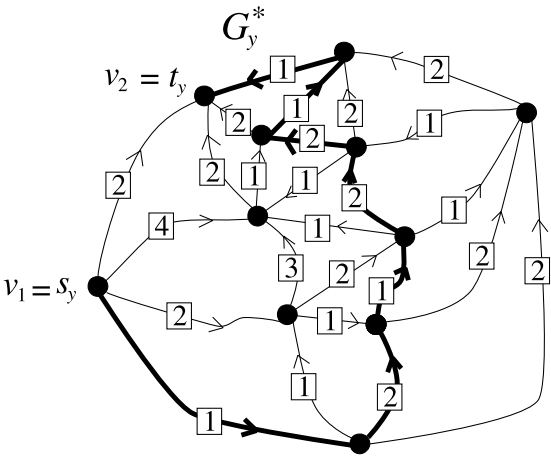

(d)

Fig. 12 (a) Directed graph $G_{x}^{*}$, (b) directed graph $G_{y}^{*}$, (c) weights $w_{x}(e)$ of edges $e$ in $G_{x}^{*}$, and (d) weights $w_{y}(e)$ of edges $e$ in $G_{y}^{*}$

direct the edges of an inner facial 3-cycle $F_{2}=v_{2} v_{3} v_{4}$ sharing an edge with $F_{1}$ for $G_{x}^{*}$ and $G_{y}^{*}$. For example, if we direct each edge of $F_{1}=v_{1} v_{2} v_{3}$ as illustrated in Fig. 14(a), then we decide the position of $v_{4}$ relatively to those of $v_{2}$ and $v_{3}$, that is, we decide the directions of edges $\left(v_{2}, v_{4}\right)$ and $\left(v_{3}, v_{4}\right)$ in $G_{x}^{*}$ and $G_{y}^{*}$ depending on which inner angle of $F_{2}$ is labeled 0 by $L^{*}$, as illustrated in Fig. 15. Repeating the operation above for each inner facial 3-cycle of $G^{*}$, we obtain a directed graph $G_{x}^{*}$ and $G_{y}^{*}$, as illustrated in Figs. 12(a) and (b). It should be noted that one can obtain the orientation by the following two rules: (i) the three edges of an inner face cannot have the same slope with respect to the sign, and (ii) a vertex $w$ with the inner angle in a face $F$ labeled 0 is simultaneously (left, right)most and (top, bottom) most among the three vertices on the face $F$, i.e., it has out-degree either 0 or 2 on $F$ in $G_{x}^{*}$ and $G_{y}^{*}$. We will show in Sect. 5, by Lemma 4 and Lemma 2, that both $G_{x}^{*}$ and $G_{y}^{*}$ are acyclic, that each of $G_{x}^{*}$ and $G_{y}^{*}$ has exactly one vertex of in-degree zero, called the source, and every other vertex has in-degree one or more, and that each of $G_{x}^{*}$ and $G_{y}^{*}$ has exactly one vertex of out-degree zero, called the sink, and every other vertex has out-degree one or more. 
Fig. 13 Three cases of the labeling of the starting inner facial cycle $F_{1}$
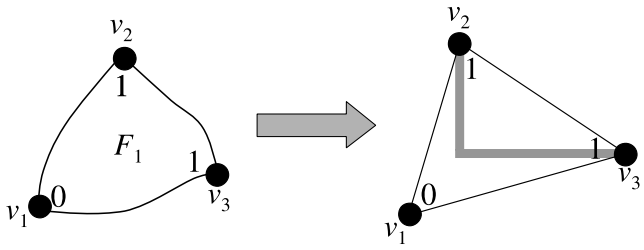

(a)
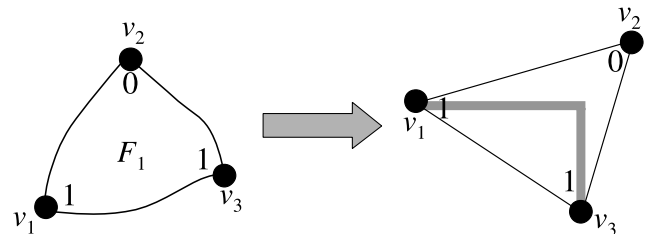

(b)
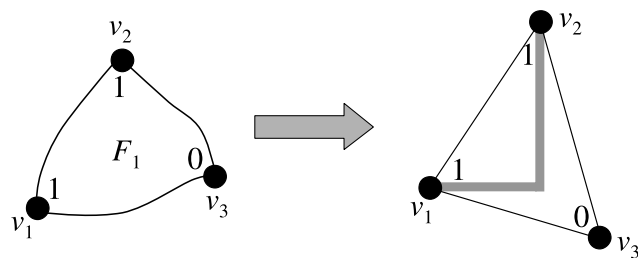

(c)

Step 2 For each edge $e=u \rightarrow v$ of $G_{x}^{*}$, we assign a positive integer weight $w_{x}(e)$ to $e$, as illustrated in Fig. 12(c) where $w_{x}(e)$ is written in a square. The weight $w_{x}(e)$ implies that $x(u)+w_{x}(e) \leq x(v)$ in $D^{*}$. We decide $w_{x}(e)$ as follows. If an inner facial cycle $F$ of $G^{*}$ is not filled in $G$, then we give, as a weight $w_{x}(e)$, either 1 or 2 to each edge $e$ of $F ; w_{x}\left(v_{1} \rightarrow v_{2}\right)=w_{x}\left(v_{2} \rightarrow v_{3}\right)=1$ and $w_{x}\left(v_{1} \rightarrow v_{3}\right)=2$ if the angle at $v_{1}$ has label 0 , as illustrated in Fig. 14(a). If $F$ is filled in $G$, that is, $F=C_{i}$ for some index $i, 1 \leq i \leq k$, then we assign a weight $w_{x}(e)$ to each edge $e$ of $F$, as illustrated in Fig. 14(b); the value $w_{x}(e)$ depends on both the number $n_{F}$ of vertices in the inside graph $G(F)$ and the index $c$ in Sect. 3; $w_{x}\left(v_{1} \rightarrow v_{2}\right)=1$, $w_{x}\left(v_{1} \rightarrow v_{3}\right)=n_{F}-c$, and $w_{x}\left(v_{2} \rightarrow v_{3}\right)=n_{F}-c-1$ if the angle at $v_{1}$ has label 0 . Since each inner edge $e$ receives two weights from the two facial cycles containing $e$, we assign $e$ the larger one as $w_{x}(e)$. Similarly, we assign a positive integer weight $w_{y}(e)$ to each edge $e$ of $G_{y}^{*}$, as illustrated in Fig. 12(d).

Step 3 Let $s_{x}$ be the source of $G_{x}^{*}$, that is, the vertex having in-degree zero. We decide the $x$-coordinate $x\left(s_{x}\right)$ of $s_{x}$ as $x\left(s_{x}\right)=0$. Since $G_{x}^{*}$ is acyclic and every vertex $u$ other than $s_{x}$ has in-degree one or more, one can find in linear time the longest path from $s_{x}$ to each vertex $u$ in $G_{x}^{*}$. We decide $x(u)$ to be the length of the longest path. Similarly, we compute the $y$-coordinate of every vertex. Thus we obtain a drawing $D^{*}$ of $G^{*}$, as illustrated in Fig. 7(b).

We will verify the correctness of our algorithm in Sect. 5.

By Theorem 1 one can find open RI-drawings $D_{i}$ of $G\left(C_{i}\right), 1 \leq i \leq k$, in linear time. From a given good labeling $L^{*}$ of $G^{*}$, one can find in linear time a good RIdrawing $D^{*}$ of $G^{*}$ realizing $L^{*}$; each of Steps $1-3$ can be done in linear time. The triangular drawing of $C_{i}, 1 \leq i \leq k$, in $D^{*}$ is large enough for $D_{i}$ so that one can 


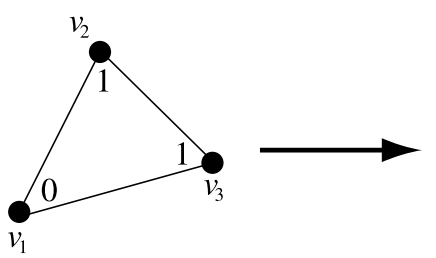

(a)

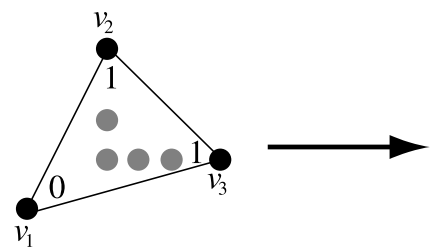

(b)

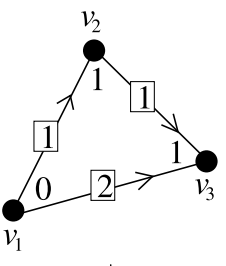

$G_{x}^{*}$

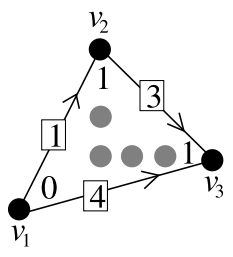

$G_{x}^{*}$

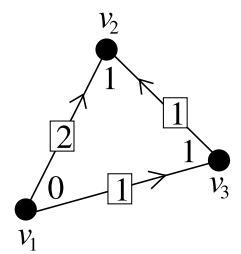

$G_{y}^{*}$

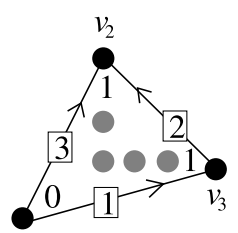

$G_{y}^{*}$

Fig. 14 Directions and weights $w_{x}(e)$ of edges $e$ in $G_{x}^{*}$ and $G_{y}^{*} ;$ (a) non-filled 3-cycle $C$, and (b) filled 3-cycle $C$ with $c=3$

embed $D_{i}$ in $D^{*}$ after enlarging $D_{i}$ if necessary. One can thus construct a good open RI-drawing $D$ of $G$ from $L^{*}$ in linear time.

Let $s_{x}$ and $t_{x}$ be the source and sink of the acyclic graph $G_{x}^{*}$, respectively. (See Lemma 4 in Sect. 5.) Let $P_{x}$ be the longest path in $G_{x}^{*}$ from $s_{x}$ to $t_{x}$ of $G_{x}^{*}$. Then

$$
x(u)+w_{x}(e)=x(v)
$$

for every edge $e=u \rightarrow v$ in $P_{x}$. Let $P_{y}$ be the longest path in $G_{y}^{*}$ from the source $s_{y}$ to the sink $t_{y}$ of $G_{y}^{*}$. Then

$$
y(u)+w_{y}(e)=y(v)
$$

for every edge $e=u \rightarrow v$ in $P_{y}$. ( $P_{x}$ and $P_{y}$ are drawn by thick lines in Figs. 12(c) and (d), respectively.) Let length $\left(P_{x}\right)$ and length $\left(P_{y}\right)$ be the length of $P_{x}$ and $P_{y}$, respectively. Then the drawing $D$ of $G$ obtained by our algorithm is on a $W \times H$ integer grid such that $W=$ length $\left(P_{x}\right)$ and $H=$ length $\left(P_{y}\right)$. By (1) and (2) there is one or more vertices of $G$ on each grid line, and hence $W, H \leq n-1$.

Thus we have the following theorem.

Theorem 3 One can construct a good open RI-drawing of an inner triangulated plane graph $G$ on a $W \times H \leq(n-1) \times(n-1)$ grid in linear time if a good labeling of $G^{*}$ is given. 

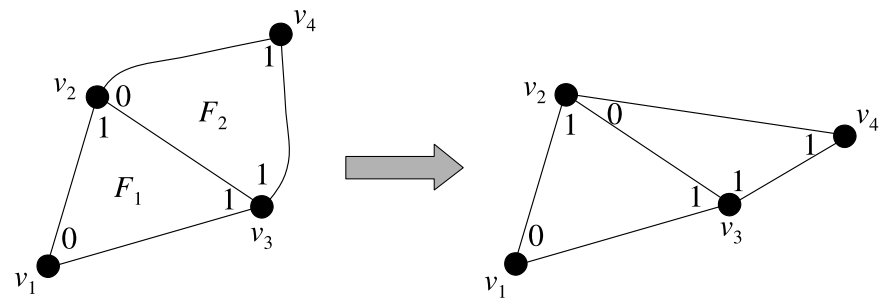

(a)
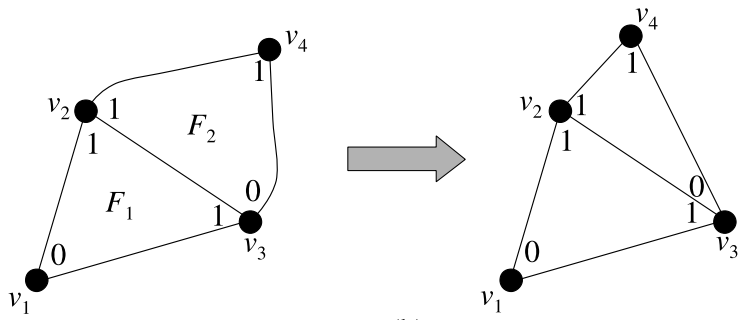

(b)
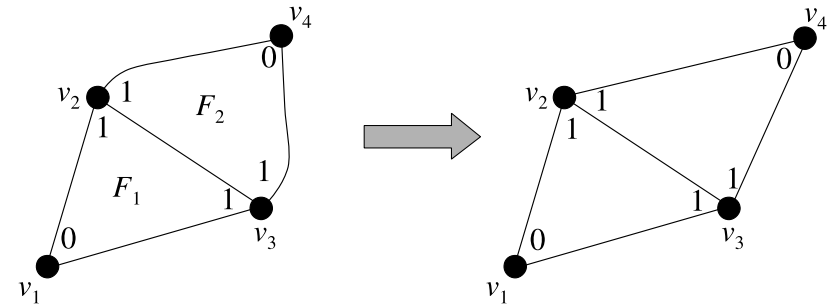

(c)

Fig. 15 A relative position of vertex $v_{4}$ in the second inner facial cycle $F_{2}=v_{2} v_{3} v_{4}$

\section{Proof of Theorem 2}

In this section, we prove Theorem 2.

Suppose that $G^{*}$ has a good labeling $L^{*}$. It suffices to prove that the drawing $D^{*}$ of $G^{*}$ obtained by Steps $1-3$ in Sect. 4.2 is an oblique RI-drawing of $G^{*}$ and realizes $L^{*}$, because one can embed in $D^{*}$ the drawing $D_{i}$ of the inside graph $G\left(C_{i}\right)$ after adjusting the size of $D_{i}$ to the triangular drawing of the maximal filled 3-cycle $C_{i}$ in $D^{*}$ for each index $i, 1 \leq i \leq k$.

For an inner facial cycle $F$ of $G^{*}$, we denote by $G_{x}^{*}(F)$ the directed subgraph of $G_{x}^{*}$ induced by $F$. Similarly $G_{y}^{*}(F)$ is defined. We then have the following lemma.

Lemma 2 The drawing $D^{*}$ obtained by Steps $1-3$ is oblique and realizes a good labeling $L^{*}$ of $G^{*}$.

Proof The weight $w_{x}(e)$ of every edge $e=u \rightarrow v$ in $G_{x}^{*}$ is positive, and $x(u)+$ $w_{x}(e) \leq x(v)$. Therefore, every edge $(u, v)$ is not vertical in $D^{*}$. Similarly every 
edge $(u, v)$ is not horizontal in $D^{*}$. Thus $D^{*}$ is oblique. Hence, it suffices to prove that $D^{*}$ realizes $L^{*}$.

Let $f_{I}$ be the number of inner faces of $G^{*}$, and let $F_{1}, F_{2}, \ldots, F_{f_{I}}$ be the inner faces of $G^{*}$. We assume that Step 1 is executed for inner faces $F_{1}, F_{2}, \ldots, F_{f_{I}}$ in this order. We indeed decide the order as follows. For each index $i, 1 \leq i \leq f_{I}$, we denote by $G_{i}^{*}$ the subgraph of $G^{*}$ induced by the edges contained in $F_{1}, F_{2}, \ldots, F_{i}$. Then $G_{f_{I}}^{*}=G^{*}$, of course. Suppose that $F_{1}$ is an arbitrary inner face containing an outer edge and that we have executed Step 1 for inner faces $F_{1}, F_{2}, \ldots, F_{i}, 1 \leq i \leq f_{I}-1$. We now choose the $(i+1)$ th inner face $F_{i+1}$ as follows. If $G^{*}$ has an inner face $F$ such that $F$ is not contained in $G_{i}^{*}$ but two edges of $F$ are contained in $G_{i}^{*}$, then we choose such a face $F$ as $F_{i+1}$. Otherwise, we choose a face having exactly one edge in $G_{i}^{*}$ as $F_{i+1}$. (Thus, for each index $i, 1 \leq i \leq f_{I}, G_{i}^{*}$ is 2 -connected and the union of the regions of inner faces $F_{1}, F_{2}, \ldots, F_{i}$ does not have a "hole.")

For each $i, 1 \leq i \leq f_{I}$, we denote by $D_{i}^{*}$ the oblique drawing of $G_{i}^{*}$ in $D^{*}$ and by $L_{i}^{*}$ the labeling of (only) the inner angles of $G_{i}^{*}$ by $L^{*}$. We prove, by induction, a proposition that $D_{i}^{*}$ realizes $L_{i}^{*}$. (Remember that an oblique drawing is defined to realize a labeling if every inner angle labeled $j$ contains exactly $j$ half-lines for each $j, 0 \leq j \leq 4$.)

We first prove the proposition for $i=1$. Let $F_{1}=v_{1} v_{2} v_{3}$. In Step 1, we decide the relative positions of $v_{1}, v_{2}$, and $v_{3}$, that is, we decide the directions and weights of the three edges of $F_{1}$ in $G_{x}^{*}$ and $G_{y}^{*}$ so that the (tentative) drawing of $G_{1}^{*}\left(=G^{*}\left(F_{1}\right)\right)$ realizes $L_{1}^{*}$, as illustrated in Figs. 13 and 14. The exact positions of $v_{1}, v_{2}$, and $v_{3}$ are decided in Step 3, but their relative positions remain as they are. Thus, the (final) drawing $D_{1}^{*}$ of $G_{1}^{*}$ realizes $L_{1}^{*}$, and hence the proposition holds for $i=1$.

We next assume inductively that the proposition holds for $i, 1 \leq i \leq f_{I}-1$, and we shall prove that the proposition holds for $i+1$.

There are the following two cases to consider.

Case 1: Exactly one of the three edges of $F_{i+1}$, say $e$, is contained in $G_{i}^{*}$. In this case, exactly one of the three vertices in $F_{i+1}$, say $v$, is not contained in $G_{i}^{*}$, and $v$ is not an end of $e$. Similarly as in Fig. 15, we decide the position of $v$ relatively to the positions of the ends of $e$, that is, we decide the directions of the two edges of $F_{i+1}$ other than $e$ in $G_{x}^{*}$ and $G_{y}^{*}$ so that $D_{i+1}^{*}$ realizes $L_{i+1}^{*}$.

Case 2: Exactly two edges of $F_{i+1}$ are contained in $G_{i}^{*}$. Let $u$ be the common end of the two edges, and let $F_{i+1}=u v w$. Since the angle of $u$ in $F_{i+1}$ is an inner angle of $G^{*}$, condition (b) of a good labeling implies that the label of $u$ in $F_{i+1}$ is either 0 or 1 . Thus there are the following two cases to consider.

Case $2 a$ : The label of $u$ in $F_{i+1}$ is 0 . (See Fig. 16.) In this case, one of the following (i), (ii), and (iii) holds for each of the 3 -cycles $G_{x}^{*}\left(F_{i+1}\right)$ and $G_{y}^{*}\left(F_{i+1}\right)$ :

(i) $u$ has in-degree 0

(ii) $u$ has in-degree 2 and

(iii) $u$ has in-degree 1.

We claim that (iii) does not occur for $G_{x}^{*}\left(F_{i+1}\right)$ and $G_{y}^{*}\left(F_{i+1}\right)$. We prove the claim only for $G_{x}^{*}\left(F_{i+1}\right)$, because one can similarly prove the claim for $G_{y}^{*}\left(F_{i+1}\right)$. Assume for a contradiction that $u$ has in-degree 1 in $G_{x}^{*}\left(F_{i+1}\right)$, as illustrated in Fig. 17. Then $u$ has out-degree 1 . We may assume without loss of generality that $v \rightarrow u$ and $u \rightarrow w$ 


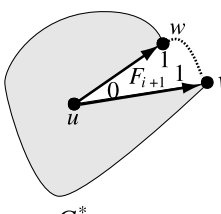

$G_{x}^{*}$

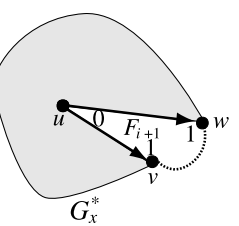

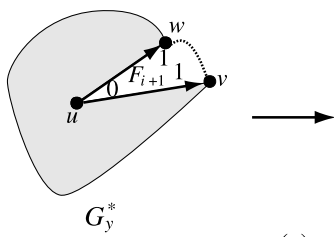

(a)

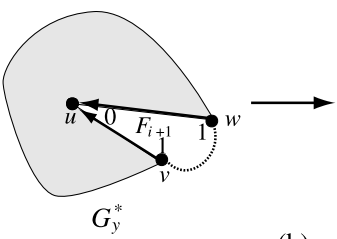

(b)
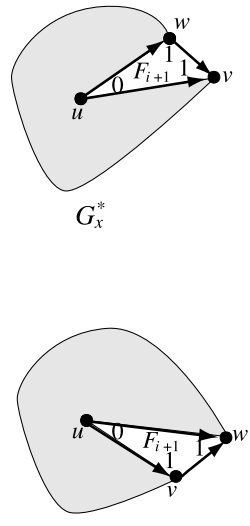

$G_{x}^{*}$
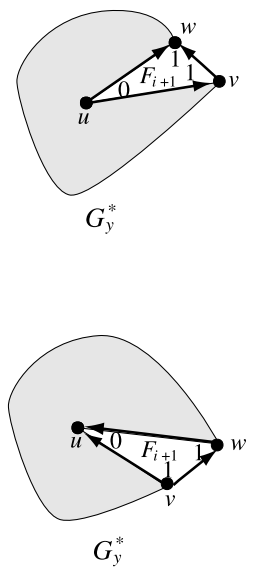

Fig. 16 Illustration for Case 2a

Fig. 17 Illustration for the case where $u$ has in-degree 1 in $G_{x}^{*}\left(F_{i+1}\right)$

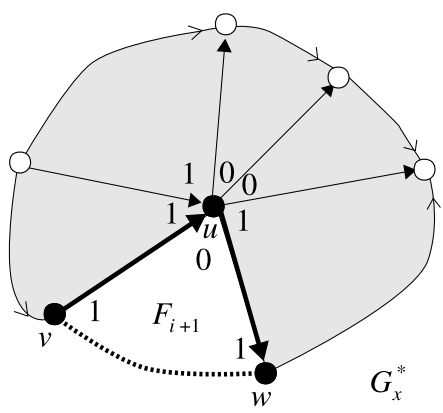

in $G_{x}^{*}$. The directions of edges $(v, u)$ and $(u, w)$ have been already decided, and $G_{i}^{*}$ is 2-connected and has no "hole." Therefore, the relative positions of all vertices around $u$ (other than the direction of edge $(v, w)$ ) have been already decided, and $u$ is an inner vertex of $G_{i+1}^{*}$. Furthermore, by the inductive assumption, the drawing $D_{i}^{*}$ of $G_{i}^{*}$ realizes a labeling $L_{i}^{*}$. Since $D_{i}^{*}$ realizes $L_{i}^{*}$ and $x(v)<x(u)$ and $x(u)<x(w)$ in $D_{i}^{*}$, the labels of angles around the inner vertex $u$ other than the angle in $F_{i+1}$ total to 1,2 , or 3 . (Figure 17 illustrates the case where the labels total to 3.) Since the label of angle of $u$ in $F_{i+1}$ is 0 , the labels of angles around $u$ total to 1,2, or 3, contrary to condition (a) of the good labeling.

Thus, for each of $G_{x}^{*}\left(F_{i+1}\right)$ and $G_{y}^{*}\left(F_{i+1}\right)$, either (i) or (ii) holds. If (i) holds for both $G_{x}^{*}\left(F_{i+1}\right)$ and $G_{y}^{*}\left(F_{i+1}\right)$, then according to the given labeling $L^{*}$ we decide the relative positions of $u, v$, and $w$, that is, we decide the directions of edge $(v, w)$ in $G_{x}^{*}$ and $G_{y}^{*}$, as illustrated in Fig. 16(a). If (i) holds for $G_{x}^{*}\left(F_{i+1}\right)$ and (ii) holds for $G_{y}^{*}\left(F_{i+1}\right)$, then we decide the directions of $(v, w)$ in $G_{x}^{*}$ and $G_{y}^{*}$, as illustrated in Fig. 16(b). For the other cases, we decide the directions of $(v, w)$ in $G_{x}^{*}$ and $G_{y}^{*}$ as similarly as above. (They can be obtained using rotations by 90,180 , and 270 degrees.) In any case, we orient the edge $(v, w)$ as that a sign of its slope in $D_{i+1}^{*}$ is 


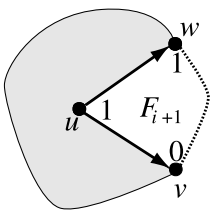

$G_{x}^{*}$

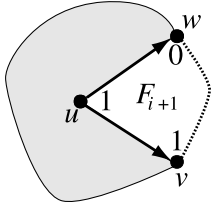

$G_{x}^{*}$

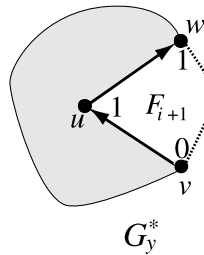

$G_{y}^{*}$
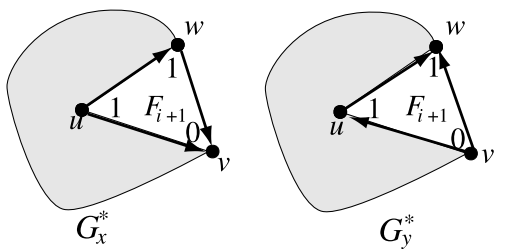

(a)
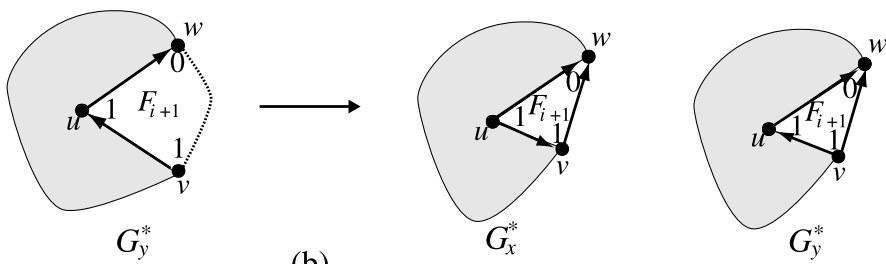

Fig. 18 Illustration for Case $2 b$

different from the sign of the slopes of $(u, v)$ and $(u, w)$. In either case, the drawing $D_{i+1}^{*}$ realizes $L_{i+1}^{*}$.

Case $2 b$ : The label of $u$ in $F_{i+1}$ is 1 . (See Fig. 18.) In this case, the label of either $w$ or $v$ in $F_{i+1}$ is 0 , and edges $(u, w)$ and $(u, v)$ have different slopes with respect to the sign. Therefore, we orient the edge $(v, w)$ in $G_{x}^{*}$ and $G_{y}^{*}$ as that the vertex $w$ or $v$ with label 0 is simultaneously (left, right)most and (top, bottom)most among the vertices $u, v$, and $w$.

Consider first the case where $u$ has in-degree 0 in $G_{x}^{*}\left(F_{i+1}\right)$. (See Figs. 18(a) and (b).) In this case, the out-degree of $u$ in $G_{x}^{*}\left(F_{i+1}\right)$ is 2 , and both the in-degree and the out-degree of $u$ in $G_{y}^{*}\left(F_{i+1}\right)$ must be 1, as illustrated in Figs. 18(a) and (b). Then, we decide the directions of $(v, w)$ in $G_{x}^{*}$ and $G_{y}^{*}$ according to the labeling $L^{*}$ so that $D_{i+1}^{*}$ realizes $L_{i+1}^{*}$, as illustrated in Figs. 18(a) and (b).

Consider then the other case where $u$ has in-degree 1 or 2 in $G_{x}^{*}\left(F_{i+1}\right)$. In this case, one can similarly orient $(v, w)$ so that $D_{i+1}^{*}$ realizes $L_{i+1}^{*}$.

We have thus completed the proof of Lemma 2.

Since $D^{*}$ realizes a good labeling $L^{*}$, by the definition every inner angle labeled $j$ by $L^{*}$ contains exactly $j$ half-lines in $D^{*}$ for each $j, 0 \leq j \leq 4$. Since $D^{*}$ is oblique and $L^{*}$ satisfies conditions (a)-(c), every outer angle labeled $j$ by $L^{*}$ contains exactly $j$ half-lines for each $j, 2 \leq j \leq 4$.

We then have the following lemma.

Lemma 3 The drawing $D^{*}$ of $G^{*}$ has no edge-crossing.

Proof We may assume without loss of generality that, in Step 3, we put all vertices in order of their $y$-coordinates: let $n^{*}$ be the number of vertices in $G^{*}$, let $w_{1}, w_{2}, \ldots, w_{n^{*}}$ be the vertices of $G^{*}$, and let $y\left(w_{1}\right) \leq y\left(w_{2}\right) \leq \cdots \leq y\left(w_{n^{*}}\right)$. For 


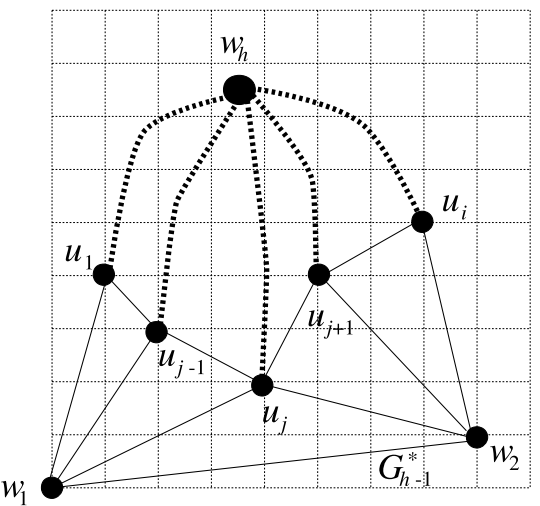

(a)

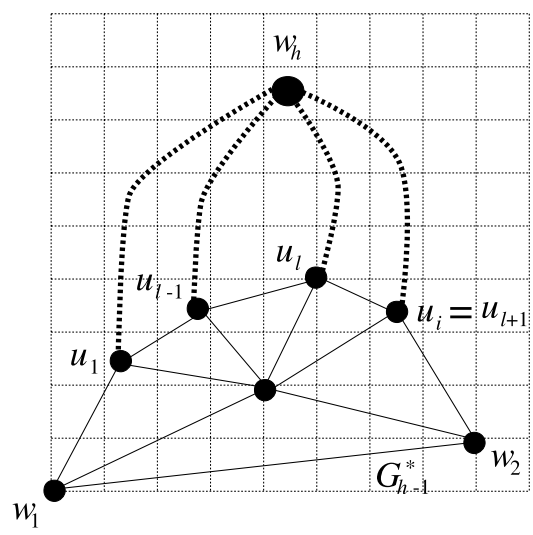

(b)

Fig. 19 Illustrations of $G_{h}^{*}$

each $h, 1 \leq h \leq n^{*}$, we denote by $G_{h}^{*}$ the subgraph of $G^{*}$ induced by vertices $w_{1}, w_{2}, \ldots, w_{h}$; then $G^{*}=G_{n^{*}}^{*}$.

Of course, $w_{1}$ is the source $s_{y}$ of $G_{y}^{*}$ and is an outer vertex. Obviously the drawing of $G_{1}^{*}$ has no edge-crossing.

Vertex $w_{2}$ has in-degree one or more in $G_{y}^{*}$, while $w_{2}$ has in-degree zero in a graph obtained from $G_{y}^{*}$ by deleting $w_{1}=s_{y}$. Therefore $G_{y}^{*}$ has an edge $w_{1} \rightarrow w_{2}$, and hence $G^{*}$ has an edge $\left(w_{1}, w_{2}\right)$. The edge $\left(w_{1}, w_{2}\right)$ is oblique in $D^{*}$, and $y\left(w_{1}\right) \leq$ $y\left(w_{2}\right) \leq y\left(w_{h}\right)$ for every $h, 3 \leq h \leq n^{*}$. Therefore, one of the two faces containing $\left(w_{1}, w_{2}\right)$ cannot have labels $0,1,1$. Thus $\left(w_{1}, w_{2}\right)$ must be an outer edge of $G^{*}$. Of course, the drawing of $G_{2}^{*}$ has no edge-crossing.

We shall prove that the drawing of $G_{h}^{*}$ has no edge-crossing for each index $h$, $3 \leq h \leq n^{*}$.

Let $u_{1}, u_{2}, \ldots, u_{i}$ be the vertices, adjacent to $w_{h}$, whose $y$-coordinates are smaller than $y\left(w_{h}\right)$. Then, noting that $D^{*}$ realizes $L^{*}$ and every vertex has degree two or more in an inner triangulated graph, one can observe that $i \geq 2$. One may assume that $u_{1}, u_{2}, \ldots, u_{i}$ appear clockwise on the outer face of the plane drawing of $G_{h-1}^{*}$ in this order. One can easily observe that $x\left(u_{1}\right)<x\left(u_{2}\right)<\cdots<x\left(u_{i}\right)$, because $D^{*}$ realizes $L^{*}$ and hence each inner face has labels $0,1,1$. Let $u_{j}, 1 \leq j \leq i$, be the vertex having the smallest $y$-coordinate among $u_{1}, u_{2}, \ldots, u_{i}$. We then claim that $y\left(u_{1}\right)>$ $y\left(u_{2}\right)>\cdots>y\left(u_{j}\right)<\cdots<y\left(u_{i}\right)$, as illustrated in Fig. 19(a). Assume otherwise that there exists a vertex $u_{l}, 2 \leq l \leq i-1$, such that $y\left(u_{l-1}\right)<y\left(u_{l}\right)>y\left(u_{l+1}\right)$, as illustrated in Fig. 19(b). Since there is no vertical edge in the drawing $D^{*}$ obtained by our algorithm, we have $x\left(u_{l}\right) \neq x\left(w_{h}\right)$. If $x\left(u_{l}\right)<x\left(w_{h}\right)$, then the labels of the inner face $w_{h} u_{l} u_{l-1}$ would be $0,2,0$, as illustrated in Fig. 20(a), contrary to the condition (b) of the good labeling. If $x\left(u_{l}\right)>x\left(w_{h}\right)$, then the labels of the inner face $w_{h} u_{l} u_{l+1}$ would be 0,2, 0, as illustrated in Fig. 20(b), contrary to the condition (b) of the good labeling.

Since the drawing $D^{*}$ realizes a good labeling $L^{*}$, each new inner face of $G_{h}^{*}$ created when $v_{h}$ is added to $G_{h-1}^{*}$ has labels $0,1,1$. Therefore, $v_{h}$ can be located only 


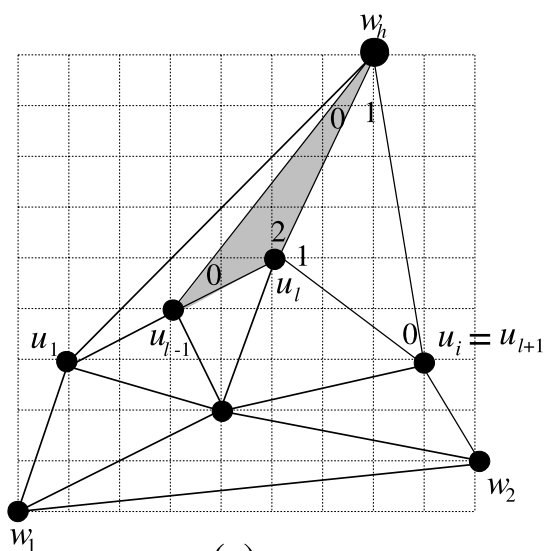

(a)

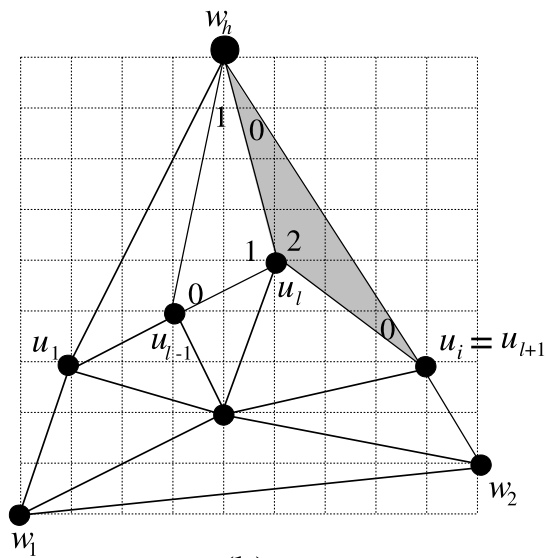

(b)

Fig. 20 Illustration of $G_{h}^{*}$ for the case where (a) $x\left(u_{l}\right)<x\left(v_{h}\right)$ and (b) $x\left(u_{l}\right)>x\left(v_{h}\right)$

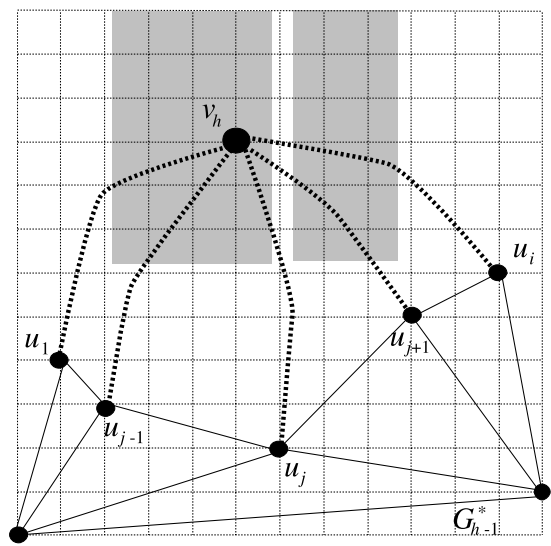

(a)

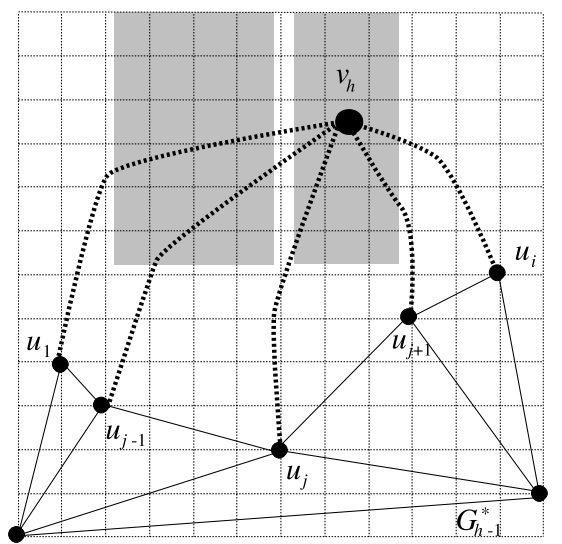

(b)

Fig. 21 Regions in which $v_{h}$ is located

in the regions shaded in Fig. 21, that is, $x\left(u_{j-1}\right)<x\left(v_{h}\right)<x\left(u_{j+1}\right), x\left(v_{h}\right) \neq x\left(u_{j}\right)$ and $\max \left\{y\left(u_{1}\right), y\left(u_{i}\right)\right\}<y\left(v_{h}\right)$. Thus, every vertex $v_{p}, 1 \leq p \leq i$, is visible from $v_{h}$, and hence no edge-crossing appears when all edges $\left(v_{h}, u_{p}\right), 1 \leq p \leq i$, are drawn. We have thus completed the proof of Lemma 3.

In order to guarantee that one can find the longest paths in $G_{x}^{*}$ and $G_{y}^{*}$, it suffices to prove the following two lemmas. 
Lemma 4 Each of $G_{x}^{*}$ and $G_{y}^{*}$ has exactly one vertex of in-degree zero, and every other vertex has in-degree one or more. Each of $G_{x}^{*}$ and $G_{y}^{*}$ has exactly one vertex of out-degree zero, and every other vertex has out-degree one or more.

Proof We prove only that $G_{x}^{*}$ has exactly one vertex of in-degree zero and that every other vertex has in-degree one or more, because the proof for the other claims is similar.

Let $n^{*}$ be the number of vertices of $G^{*}$, let $n_{o}$ be the number of outer vertices of $G^{*}$, let $m$ be the number of edges of $G^{*}$, and let $f_{I}$ be the number of inner faces of $G^{*}$. Then, by Euler's formula [8], we have

$$
n^{*}-m+f_{I}+1=2 .
$$

Since each inner facial cycle is a 3-cycle, we have

$$
2 m=3 f_{I}+n_{o}
$$

By (3) and (4), we have

$$
f_{I}=2 n^{*}-n_{o}-2 \text {. }
$$

Since the labeling $L^{*}$ satisfies condition (a) of the good labeling, the labels of all angles total to $4 n^{*}$. Furthermore, by condition (b) of the good labeling, the labels of all inner angles total to $2 f_{I}$. Thus the labels of all outer angles total to

$$
\begin{aligned}
4 n^{*}-2 f_{I} & =4 n^{*}-2\left(2 n^{*}-n_{o}-2\right) \\
& =2 n_{o}+4 .
\end{aligned}
$$

Therefore, by condition (c) of the good labeling, there are the following three cases (I)-(III).

(I) Four outer angles have label 3, and all the other $n_{o}-4$ outer angles have label 2, as illustrated in Fig. 22(a).

(II) Two outer angles have label 3, one outer angle has label 4, and all the other $n_{o}-3$ outer angles have label 2, as illustrated in Fig. 22(b).

(III) Two outer angles have label 4, and all the other $n_{o}-2$ outer angles have label 2, as illustrated in Fig. 22(c).

The outer facial cycle of $G_{x}^{*}$ can be partitioned into maximal directed paths, whose endpoints are the only candidates for the sinks and sources of $G_{x}^{*}$. Moreover, the outer angles of these endpoints can have labels only either 3 or 4 , and any such path, whose two endpoints have their outer angles labeled by 3 , has to contain third vertex having the outer angle labeled by 3 . One can thus observe that there are exactly two paths in such a partition; one goes clockwise from an outer vertex $a$ to an outer vertex $b$, and the other goes counterclockwise from $a$ to $b$. Since the label of the outer angle of $a$ is 3 or 4 , at most one of the inner angles of $a$ has label 1, and all the others have label 0 . Thus, the outer vertex $a$ has in-degree zero in $G_{x}^{*}$, and every other outer vertex has in-degree one or more. Since $D^{*}$ realizes a good labeling $L^{*}$ of $G^{*}$, one can easily observe that every inner vertex of $G_{x}^{*}$ has in-degree two or more. Thus only the outer 


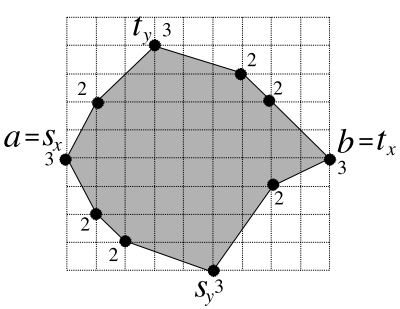

(a)

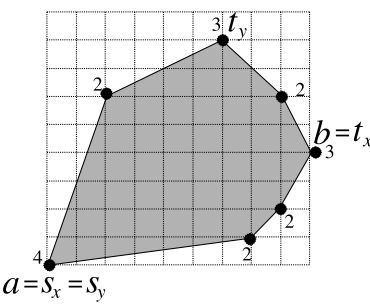

(b)

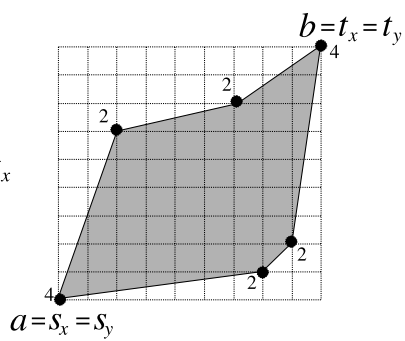

(c)

Fig. 22 (a) Outer facial cycle of Case (I), (b) Case (II), and (c) Case (III)

vertex $a$ has in-degree zero in $G_{x}^{*}$, and every other vertex has in-degree one or more. This completes the proof of Lemma 4. (Note that $a=s_{x}$ and $b=t_{x}$.)

We then have the following lemma.

Lemma 5 Both $G_{x}^{*}$ and $G_{y}^{*}$ are acyclic.

Proof We prove only that $G_{x}^{*}$ is acyclic, because one can similarly prove that $G_{y}^{*}$ is acyclic. Suppose for a contradiction that $G_{x}^{*}$ has a directed cycle $C=v_{0} v_{1} \cdots v_{p}$, where $v_{p}=v_{0}$.

By Lemmas 2 and 3, $D^{*}$ is an oblique drawing realizing a given good labeling $L^{*}$ of $G^{*}$. Therefore, we have $x(u)<x(v)$ for each directed edge $u \rightarrow v$ of $G_{x}^{*}$, and hence $x\left(v_{0}\right)<x\left(v_{1}\right)<\cdots<x\left(v_{p}\right)$. This contradicts to the assumption that $v_{p}=v_{0}$. We have thus completed the proof of Lemma 5.

Using Lemmas 2 and 3, we finally prove the following lemma.

\section{Lemma 6 The drawing $D^{*}$ is an oblique closed RI-drawing of $G^{*}$.}

Proof Suppose for a contradiction that $D^{*}$ is not a closed RI-drawing of $G^{*}$. Then there is a pair of an edge $e$ and a vertex $z$ such that $z$ is contained in a closed rectangleof-influence of $e$. One may assume without loss of generality that the distance between $e$ and $z$ is minimum among all these pairs. $G^{*}$ has two faces containing $e$. Let $F$ be the one of them which is in the same side of $e$ as $z$. If $F$ is the outer face of $G^{*}$, then $F$ has a shape illustrated in Figs. 22(a)-(c), and hence $z$ cannot be located in the rectangle-of-influence of $e$, a contradiction. Thus $F$ is an inner face of $G^{*}$. Let $e=(u, v)$ and $F=u v w$. Then vertex $w$ is not contained in the rectangle-of-influence of $e$, because, by Lemma 2, $D^{*}$ realizes the good labeling $L^{*}$ of $G^{*}$. Furthermore, $z$ is not contained in $F$, because, by Lemma 3, there is no edge-crossing in $D^{*}$. Therefore, $z$ is located in a rectangle-of-influence of $(u, w)$ or $(v, w)$, say $(u, w)$, as illustrated in Fig. 23. Then, the distance between $(u, w)$ and $z$ is smaller than that between $e$ and $z$, a contradiction. We have thus completed the proof of Lemma 6.

It should be noted that condition (c) of a good labeling is crucial in the proofs of Lemmas 4 and 6. 
Fig. 23 Edge $e$, vertex $z$,

face $F$, and the

rectangles-of-influence of $e$ and $(u, w)$

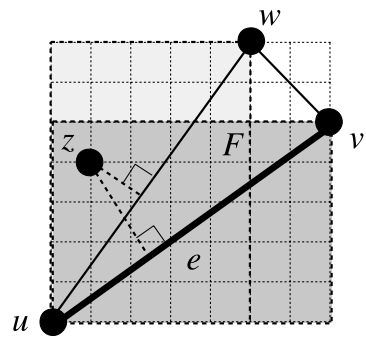

\section{Algorithm for Computing a Good Labeling}

In this section, we show how to find a good labeling of the frame graph $G^{*}$ of an inner triangulated plane graph $G$.

We assign each angle of $G^{*}$ with label $0,1, x$, or $y$, as illustrated in Fig. 24(a). The values of labels $x$ 's and $y$ 's are undecided at this moment; $x$ will be decided to be 0 or 1 , and $y$ to be 2,3, or 4. Each of the outer angles of $G^{*}$ is assigned a label $y$. Each of the inner angles is assigned a label 0,1 , or $x$ as follows. For every inner facial 3 -cycle $C$ of $G^{*}$ that is none of the maximal filled 3 -cycles $C_{1}, C_{2}, \ldots, C_{k}$ in $G$, we assign a label $x$ to each of the three angles in $C$. For every inner facial 3-cycle $C=u v w$ of $G^{*}$, that is, $C_{1}, C_{2}, \ldots$, or $C_{k}$, we assign labels as follows: if exactly one of $u, v$, and $w$, say $w$, is adjacent to all the other vertices of $G(C)$ as $C_{1}$ in Fig. 2(a), then we assign 0 to the angle of $w$ in $C$ and assign 1 to the other two angles in $C$; if exactly two, say $v$ and $w$, are adjacent to all the other vertices of $G(C)$ as $C_{2}$ in Fig. 2(a), then we assign label $x$ to the angles of $v$ and $w$ in $C$ and assign 1 to the other; if each of $u, v$, and $w$ is adjacent to all the other vertices of $G(C)$ as $C_{3}$ in Fig. 2(a), then $G(C)=K_{4}$, each of $u, v$, and $w$ can have label 0 , and hence we assign a label $x$ to the three angles in $C$. This completes the labeling of $G^{*}$ by $0,1, x$, and $y$. Our problem is to determine all the values of $x$ 's and $y$ 's so that the resulting labeling is a good labeling of $G^{*}$, that is, it satisfies conditions (a), (b), and (c) in Sect. 4.1.

\subsection{Reduction to an $f$-factor Problem}

In this subsection, we show that the problem above can be reduced to an " $f$-factor" problem on a new graph $G_{f}^{*}$, where $f$ is a function from the set $V\left(G_{f}^{*}\right)$ of vertices in $G_{f}^{*}$ to nonnegative integers. An $f$-factor of $G_{f}^{*}$ is a spanning subgraph of $G_{f}^{*}$ in which each vertex $v$ has degree $f(v)$ [8].

The new graph $G_{f}^{*}$ is constructed from $G^{*}$, as illustrated in Fig. 24(b), where $G_{f}^{*}$ is drawn by solid black lines and $G^{*}$ by dotted gray lines. We include all vertices of $G^{*}$ in $G_{f}^{*}$ but do not include any edge of $G^{*}$ in $G_{f}^{*}$. If an inner facial cycle $C$ of $G^{*}$ has an angle labeled $x$, then we add a new vertex $v_{C}$ in $C$ and join $v_{C}$ with each of the vertices in $C$ labeled $x$. Furthermore, we add a new vertex $v_{o}$ in the outer face and join $v_{o}$ with each outer vertex of $G^{*}$ by a pair of multiple edges. The resulting graph is $G_{f}^{*}$.

We then define a function $f: V\left(G_{f}^{*}\right) \rightarrow\{0,1, \ldots, 4\}$ as follows. (In Fig. 24(b), $f(v)$ is attached to each vertex $v$.) 
(a) If $v=v_{C} \in V\left(G_{f}^{*}\right)$ for an inner facial cycle $C$ of $G^{*}$, then let

$$
f\left(v_{C}\right)=2-n_{C},
$$

where $n_{C}$ is the number of inner angles in $C$ which are labeled 1 . This means that exactly the number $f\left(v_{C}\right)$ of the labels $x$ 's in $C$ must be 1 so that exactly two of the three labels in $C$ are 1, that is, condition (b) holds for $C$. Of course, $0 \leq n_{C} \leq 1$ and hence $1 \leq f\left(v_{C}\right) \leq 2$. If $C$ is none of the maximal filled 3 cycles $C_{1}, C_{2}, \ldots, C_{k}$ of $G$, then $n_{C}=0$ and hence $f\left(v_{C}\right)=2$. (In Fig. 24(b), $f\left(v_{C_{2}}\right)=1$ and $f\left(v_{C_{3}}\right)=2$, while there is no vertex $v_{C_{1}}$, because none of the inner angles of $C_{1}$ is labeled $x$.)

(b) If $v \in V\left(G_{f}^{*}\right)$ is an inner vertex of $G^{*}$, then let

$$
f(v)=4-n_{1},
$$

where $n_{1}$ is the number of inner angles around $v$ which are labeled 1 . This means that exactly $f(v)$ of the labels $x$ 's around $v$ must be 1 so that exactly four of the labels around $v$ are 1 , that is, condition (a) holds for $v$. If $n_{1} \geq 5$, then $G$ has no open RI-drawing. Therefore, one may assume that $n_{1} \leq 4$ and hence $0 \leq f(v) \leq 4$.

(c) If $v \in V\left(G_{f}^{*}\right)$ is an outer vertex of $G^{*}$, then let

$$
f(v)=2-n_{1},
$$

where $n_{1}$ is the number of inner angles around $v$ which are labeled 1 . This means that

$$
f(v)=y^{\prime}+n_{x=1}
$$

and hence $n_{1}+n_{x=1}+y=4$ and condition (a) holds for $v$, where $y$ is the label of the outer angle of $v, y^{\prime}=y-2$, and $n_{x=1}$ is the number of labels $x$ 's around $v$ that must be 1 's. One may assume that $0 \leq y^{\prime} \leq 2,0 \leq n_{1} \leq 2$, and hence $0 \leq f(v) \leq 2$; otherwise, $G$ has no good open RI-drawing.

(d) If $v=v_{o} \in V\left(G_{f}^{*}\right)$, then let

$$
f\left(v_{o}\right)=4
$$

This means that the sum of the labels $y$ of all outer angles must be $2 n_{o}+4$ (see (3) in Sect. 6), and hence the sum of $y^{\prime}=y-2$ taken over all outer angles must be 4 , where $n_{o}$ is the number of outer vertices of $G^{*}$.

One can easily observe that $G^{*}$ has a good labeling, as illustrated in Fig. 24(d), if and only if $G_{f}^{*}$ has an $f$-factor, as illustrated in Fig. 24(b) by thick solid lines. Note that an edge $e$ in an $f$-factor corresponds to an inner angle of label $x=1$ if $e$ is not incident to $v_{o}$ in $G_{f}^{*}$ and that if exactly $y^{\prime}$ of the two multiple edges joining $v_{o}$ and an outer vertex $v$ of $G^{*}$ are contained in an $f$-factor, then the label $y$ of $v$ is $y^{\prime}+2$. 


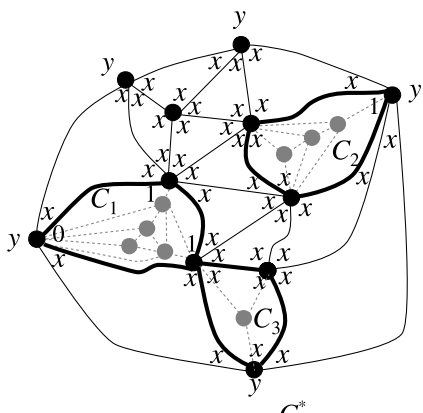

(a)

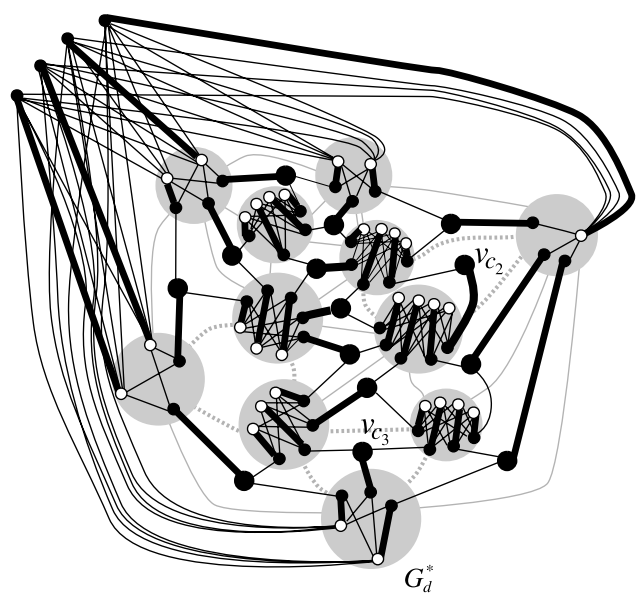

(c)

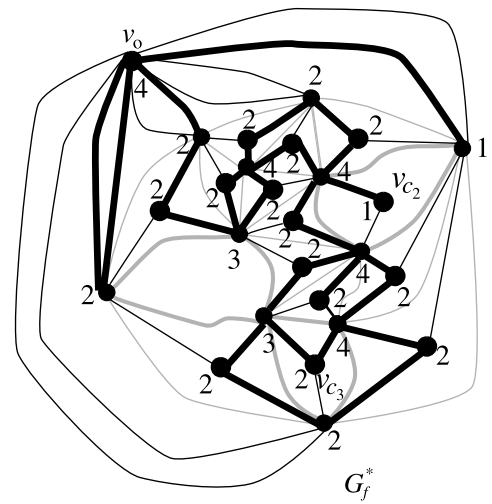

(b)

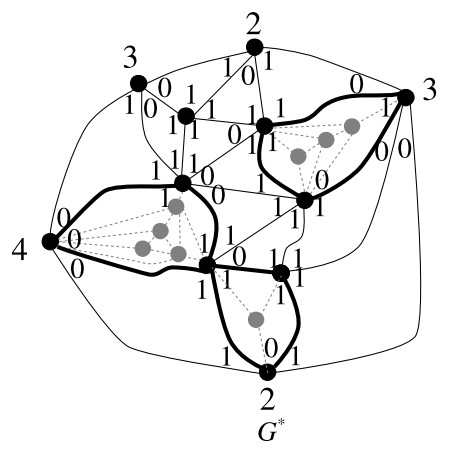

(d)

Fig. 24 (a) A labeling of $G^{*}$ with $0,1, x$, and $y,(\mathbf{b})$ a graph $G_{f}^{*}$ with an $f$-factor drawn by thick solid lines, (c) a decision graph $G_{d}^{*}$ with a perfect matching drawn by thick solid lines, and (d) a good labeling of $G^{*}$

\subsection{Reduction to a Perfect Matching Problem}

In this subsection, we show that the $f$-factor problem on $G_{f}^{*}$ above can be reduced to a "perfect matching problem" on a new graph $G_{d}^{*}$ constructed from $G_{f}^{*}$ and $f$, as illustrated in Fig. 24(c). We will show that $G_{f}^{*}$ has an $f$-factor if and only if $G_{d}^{*}$ has a perfect matching. A perfect matching of $G_{d}^{*}$ is drawn by thick lines in Fig. 24(c).

We now show how to construct a decision graph $G_{d}^{*}$. We include in $G_{d}^{*}$ a vertex $v_{C}$ for each inner facial cycle $C$ of $G^{*}$ such that at least one of the three inner angles of $C$ is labeled $x$. We add to $G_{d}^{*}$ a complete bipartite graph for each vertex $v \in$ $V\left(G_{f}^{*}\right)$ corresponding to an original vertex of $G^{*}$ as follows. Let $n_{x}$ be the number of inner angles around vertex $v$ of $G^{*}$ labeled $x$, and let $\alpha_{1}, \alpha_{2}, \ldots, \alpha_{n_{x}}$ be these angles. (See Fig. 25.) We add to $G_{d}^{*}$ the vertices $v_{1}, v_{2}, \ldots, v_{n_{x}}$ corresponding to the angles 
Fig. 25 Construction of $G_{d}^{*}$ around an inner vertex $v$ of $G^{*}$, where $n_{x}=4, n_{1}=1$, and edges in a perfect matching are drawn by thick lines

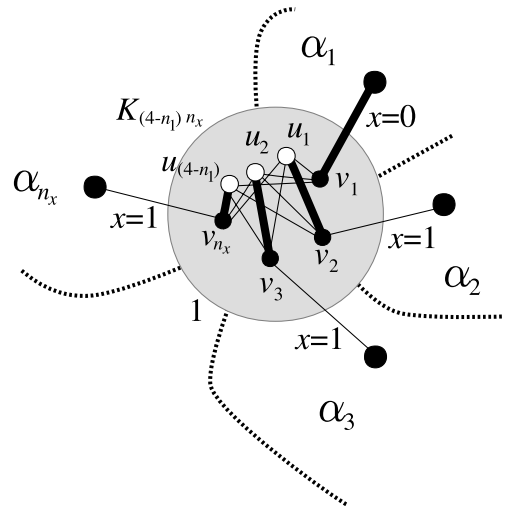

$\alpha_{1}, \alpha_{2}, \ldots, \alpha_{n_{x}}$. If an angle $\alpha_{i}, 1 \leq i \leq n_{x}$, is in an inner face $C$, then we join $v_{C}$ and $v_{i}$ by an edge. Let $n_{1}$ be the number of inner angles around vertex $v$ of $G^{*}$ which have been labeled 1 . One may assume that $n_{1} \leq 4$; otherwise, $G$ has no RI-drawing. If $v$ is an inner vertex of $G^{*}$, then we add $4-n_{1}$ new vertices $u_{1}, u_{2}, \ldots, u_{4-n_{1}}$ to $G_{d}^{*}$; we then join each of the $4-n_{1}$ vertices $u_{1}, u_{2}, \ldots, u_{4-n_{1}}$ with all the $n_{x}$ vertices $v_{1}, v_{2}, \ldots, v_{n_{x}}$ so that a complete bipartite graph $K_{\left(4-n_{1}\right) n_{x}}$ would be a subgraph of $G_{d}^{*}$, as illustrated in Fig. 25. If $v$ is an outer vertex of $G^{*}$, then, by condition (c) of the good labeling, we may assume that $n_{1} \leq 2$, and we add new $2-n_{1}$ vertices, which correspond to the outer angle of $v$; we then join each of them with all the $n_{x}$ vertices $v_{1}, v_{2}, \ldots, v_{n_{x}}$ so that a complete bipartite graph $K_{\left(2-n_{1}\right) n_{x}}$ is a subgraph of $G_{d}^{*}$. We finally add to $G_{d}^{*}$ four vertices in place of $v_{o}$ and join each of them with all the $2-n_{1}$ vertices corresponding to each outer vertex of $G^{*}$ so that a complete bipartite graph $K_{n_{o s} 4}$ is a subgraph of $G_{d}^{*}$, where $n_{o s}$ is the sum of $2-n_{1}$ taken over all outer vertices $v$ of $G^{*}$. The resulting graph is our decision graph $G_{d}^{*}$. (Tutte showed how to reduce an $f$-factor problem to a perfect matching problem of a new graph [19]. Our construction of $G_{d}^{*}$ is simpler than his construction.)

A matching of $G_{d}^{*}$ is a set of pairwise nonadjacent edges in $G_{d}^{*}$. A maximum matching of $G_{d}^{*}$ is a matching of the maximum cardinality. A matching $M$ of $G_{d}^{*}$ is called a perfect matching if an edge in $M$ is incident to each vertex of $G_{d}^{*}$.

One can easily observe that $G_{f}^{*}$ has an $f$-factor if and only if $G_{d}^{*}$ has a perfect matching. If an edge $e$ of $G_{d}^{*}$ corresponding to an inner angle labeled $x$ is contained in a perfect matching $M$ of $G_{d}^{*}$, then $x=0$. Conversely, if $e$ is not contained in $M$, then $x=1$. (See Fig. 25.) Exactly one of the labels $x$ 's in each inner face $C$ of $G^{*}$ must be 0 , and hence exactly one edge incident to $v_{C}$ must be contained in $M$. If exactly $y^{\prime}$ edges in $M$ are incident to the $2-n_{1}$ vertices corresponding to the outer angle of an outer vertex $v$, then $y=y^{\prime}+2$ for the label $y$ of $v$. (See Figs. 24(c) and (d).)

If $G$ has a good open RI-drawing, then $G^{*}$ has a good labeling and hence $G_{d}^{*}$ has a perfect matching. Conversely, if $G_{d}^{*}$ has a perfect matching, then $G^{*}$ has a good labeling and hence $G$ has a good open RI-drawing. Thus $G$ has a good open RI-drawing if and only if $G_{d}^{*}$ has a perfect matching. 


\subsection{Theorem}

Since $G_{d}^{*}$ is a bipartite graph and has $O(n)$ vertices and edges, one can determine in time $O\left(n^{1.5} / \log n\right)$ whether $G_{d}^{*}$ has a perfect matching $[9,10]$.

One can construct a good labeling of $G^{*}$ from an $f$-factor of $G_{f}^{*}$ or a perfect matching of $G_{d}^{*}$ in linear time. We thus have the following theorem.

Theorem 4 For an inner triangulated plane graph $G$, one can determine whether $G^{*}$ has a good labeling and, if so, one can compute a good labeling $L^{*}$ of $G^{*}$ in time $O\left(n^{1.5} / \log n\right)$. From $L^{*}$ one can construct a good open RI-drawing of $G$ on an $(n-1) \times(n-1)$ grid in linear time.

\section{Conclusions}

In this paper, we first showed how to find an open RI-drawing of a triangulated plane graph in linear time. We then obtained a sufficient condition for an inner triangulated plane graph $G$ to have an open RI-drawing. Indeed, it is a necessary and sufficient condition to have a good open RI-drawing: $G$ has a good open RI-drawing if and only if the frame graph $G^{*}$ of $G$ has a good labeling. We finally presented an algorithm to construct a good open RI-drawing of $G$ on an $(n-1) \times(n-1)$ grid in time $O\left(n^{1.5} / \log n\right)$ whenever $G$ has one. It would be interesting to know whether the complexity can be improved or not. In the case where $G$ has no filled 3-cycle, our algorithm provides a closed RI-drawing of $G$. It is an alternative algorithm to the algorithm of Biedl et al. [1] for the family of plane graphs with no filled 3-cycle.

It is an open problem to characterize a plane graph having an open RI-drawing, which is not necessarily inner triangulated. In general, a planar graph may have many embeddings. Thus, it is also an open problem to find an embedding of a given planar graph which admits an open RI-drawing.

Acknowledgements We thank Yuusuke Ogaki for fruitful discussions and the anonymous referees who provided us many comments which were useful to improve the presentation of the paper.

\section{References}

1. Biedl, T., Bretscher, A., Meijer, H.: Rectangle of influence drawings of graphs without filled 3-cycles. In: Proc. of Graph Drawing 1999. LNCS, vol. 1731, pp. 359-368. Springer, Berlin (2000)

2. Biedl, T., Kant, G., Kaufmann, M.: On triangulating planar graphs under the four-connectivity constraint. Algorithmica 19(4), 427-446 (1997)

3. Chrobak, M., Kant, G.: Convex grid drawings of 3-connected planar graphs. Int. J. Comput. Geom. Appl. 7(3), 211-223 (1997)

4. Di Battista, G., Eades, P., Tamassia, R., Tollis, I.G.: Graph Drawing: Algorithms for the Visualization of Graphs. Prentice-Hall, New York (1999)

5. Di Battista, G., Lenhart, W., Liotta, G.: Proximity drawability: A survey. In: Proc. of Graph Drawing 1994. LNCS, vol. 894, pp. 328-339. Springer, Berlin (1995)

6. de Fraysseix, H., Pach, J., Pollack, R.: How to draw a graph on a grid. Combinatorica 10, 41-51 (1990) 
7. Fusy, É: Transversal structures on triangulations: combinatorial study and straight-line drawing. In: Proc. of 13th International Symposium on Graph Drawing 2005 (GD 2005). LNCS, vol. 3843, pp. 177-188. Springer, Berlin (2006)

8. Harary, F.: Graph Theory. Addison-Wesley, Reading (1969)

9. Hochbaum, D.S.: Faster pseudoflow-based algorithms for the bipartite matching and the closure problems. In: CORS/SCRO-INFORMS Joint Int. Meeting, Banff, Canada, p. 46, 16-19 May 2004

10. Hochbaum, D.S., Chandran, B.G.: Further below the flow decomposition barrier of maximum flow for bipartite matching and maximum closure. Working paper (2004)

11. Jaromczyk, J.W., Toussaint, G.T.: Relative neighborhood graphs and their relatives. Proc. IEEE 80, 1502-1517 (1992)

12. Kant, G., He, X.: Regular edge-labeling of 4-connected plane graphs and its applications in graph drawing problems. Theor. Comput. Sci. 172, 175-193 (1997)

13. Liotta, G., Lubiw, A., Meijer, H., Whitesides, S.H.: The rectangle of influence drawability problem. Comput. Geom. Theory Appl. 10(1), 1-22 (1998)

14. Miura, K., Matsuno, T., Nishizeki, T.: Open rectangle-of-influence drawings of inner triangulated plane graphs. In: Proc. of 14th International Symposium on Graph Drawing 2006 (GD 2006). LNCS, vol. 4372, pp. 138-149. Springer, Berlin (2007)

15. Miura, K., Nishizeki, T.: Rectangle-of-influence drawings of four-connected plane graphs. In: Proc. of Information Visualisation 2005, Asia-Pacific Symposium on Information Visualisation (APVIS2005). ACS, vol. 45, pp. 71-76 (2005)

16. Miura, K., Nakano, S., Nishizeki, T.: Grid drawings of four-connected plane graphs. Discrete Comput. Geom. 26(1), 73-87 (2001)

17. Nishizeki, T., Rahman, M.S.: Planar Graph Drawing. World Scientific, Singapore (2004)

18. Schnyder, W.: Embedding planar graphs in the grid. In: Proc. 1st Annual ACM-SIAM Symp. on Discrete Algorithms, San Francisco, pp. 138-147 (1990)

19. Tutte, W.T.: A short proof of the factor theorem for finite graphs. Can. J. Math. 6, 347-352 (1954) 